\title{
Angiogenesis stimulated by elevated PDGF-BB in subchondral bone contributes to osteoarthritis development
}

\author{
Weiping Su, ${ }^{1,2}$ Guanqiao Liu, ${ }^{1,3}$ Xiaonan Liu, ${ }^{1,3}$ Yangying Zhou, ${ }^{4}$ Qi Sun, ${ }^{1,5}$ Gehua Zhen, ${ }^{1}$ Xiao Wang, ${ }^{1}$ \\ Yihe Hu, ${ }^{2}$ Peisong Gao, ${ }^{6}$ Shadpour Demehri, ${ }^{7}$ Xu Cao, ${ }^{1}$ and Mei Wan ${ }^{1}$ \\ 'Department of Orthopaedic Surgery, The Johns Hopkins University School of Medicine, Baltimore, Maryland, USA. \\ ${ }^{2}$ Department of Orthopaedic Surgery, The Xiangya Hospital of Central South University, Changsha, China. ${ }^{3}$ Department \\ of Orthopaedics and Traumatology, Nanfang Hospital, Southern Medical University, Guangzhou, China. ${ }^{4}$ Department of \\ Oncology, The Xiangya Hospital of Central South University, Changsha, China. ${ }^{5}$ Department of Orthopaedics, Shanghai

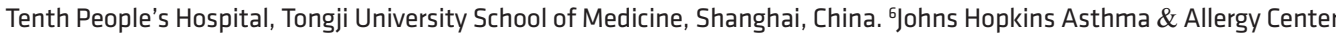 \\ and 'Department of Radiology, The Johns Hopkins University School of Medicine, Baltimore, Maryland, USA.
}

Increased subchondral bone angiogenesis with blood vessels breaching the tidemark into the avascular cartilage is a diagnostic feature of human osteoarthritis. However, the mechanisms that initiate subchondral bone angiogenesis remain unclear. We show that abnormally increased platelet-derived growth factor-BB (PDCF-BB) secretion by mononuclear preosteoclasts induces subchondral bone angiogenesis, contributing to osteoarthritis development. In mice after destabilization of the medial meniscus (DMM), aberrant joint subchondral bone angiogenesis developed during an early stage of osteoarthritis, before articular cartilage damage occurred. Mononuclear preosteoclasts in subchondral bone secrete excessive amounts of PDCF-BB, which activates platelet-derived growth factor receptor- $\beta$ (PDGFR- $\beta$ ) signaling in pericytes for neovessel formation. Selective knockout of PDGF-BB in preosteoclasts attenuates subchondral bone angiogenesis and abrogates joint degeneration and subchondral innervation induced by DMM. Transgenic mice that express PDGF-BB in preosteoclasts recapitulate pathological subchondral bone angiogenesis and develop joint degeneration and subchondral innervation spontaneously. Our study provides the first evidence to our knowledge that PDGF-BB derived from preosteoclasts is a key driver of pathological subchondral bone angiogenesis during osteoarthritis development and offers a new avenue for developing early treatments for this disease.

Conflict of interest: The authors have declared that no conflict of interest exists.

Copyright: ( 2020 , American Society for Clinical Investigation.

Submitted: December 3, 2019

Accepted: March 18, 2020

Published: April 23, 2020

Reference information: /CI Insight. 2020;5(8):e135446.

https://doi.org/10.1172/jici.

insight.135446.

\section{Introduction}

Osteoarthritis is the most prevalent chronic joint disease affecting knees, hands, hips, and spine; it is one of the leading musculoskeletal causes of impaired mobility (1-3). Currently, no effective disease-modifying drug is available to treat osteoarthritis (4-6) mainly because of the limited understanding of the mechanisms that drive the pathological process at the initiation stage. Osteoarthritis is characterized by progressive degeneration of articular cartilage (AC), structural alterations of subchondral bone, osteophyte formation, and synovial inflammation $(3,7,8)$. AC degeneration, the primary concern in osteoarthritis that leads to joint pain and dysfunction, was initially thought to be the only factor driving osteoarthritis development (9-11). However, treatments targeting only the signaling mechanisms responsible for AC degeneration may be insufficient to halt disease progression (7, 12-14). Recent evidence suggests that pathological alterations in subchondral bone also contribute to osteoarthritis development (15-23).

$\mathrm{AC}$ and subchondral bone are integrated through the osteochondral junction, which consists of the calcified cartilage zone and subchondral plate underneath. This structure allows AC and subchondral bone to act in concert as one functional unit $(8,18)$. Bone provides mechanical support for the overlying AC during joint movement and undergoes constant adaptation (modeling and remodeling) in response to changes in the mechanical environment. Changes in the subchondral bone microarchitecture precede AC damage in osteoarthritis in humans (24-27). Specifically, in early-stage osteoarthritis, the bone remodeling rate is up to 20-fold faster relative to normal bone, and markers of bone remodeling, such as osteoclast activity, are increased. The rapid 
subchondral bone turnover observed in osteoarthritis leads to changes in the bone marrow microenvironment and simultaneous neovascularization. Increased subchondral bone angiogenesis, with blood vessel invasion into the avascular cartilage, is an early diagnostic feature of human osteoarthritis (3, 28-31). This osteochondral angiogenesis not only stimulates early osteophyte development and ossification in the cartilage but also causes innervation of AC, causing joint pain. Consistently, animal studies have shown that aberrant subchondral bone angiogenesis coupled with osteogenesis may contribute to the development of subchondral bone marrow lesions, increased subchondral bone plate thickness, and eventual AC damage (16, 32-35). However, the key factor(s) for the development of pathological subchondral bone angiogenesis and the main source of the factor(s) during osteoarthritis development remain unclear.

Increases in osteoclast activity and turnover rate in subchondral bone in response to aberrant mechanical loading are often among the first detectable osteoarthritis alterations (16, 36-38). Osteoclasts are derived from bone marrow monocytes/macrophages. Under physiological conditions, monocytes/macrophages first commit to macrophage colony-stimulating factor receptor-positive osteoclast precursor cells and then differentiate into receptor activator of nuclear factor $\kappa \mathrm{B}$-positive $\left(\mathrm{RANK}^{+}\right)$tartrate-resistant acid phosphatase $\left(\mathrm{TRAP}^{+}\right)$mononuclear preosteoclasts and eventually fuse to form mature multinuclear osteoclasts (39-45). Serving as precursors for osteoclasts, preosteoclasts have limited bone-resorbing activity. We previously revealed that bone/bone marrow mononuclear preosteoclasts, i.e., $\mathrm{TRAP}^{+}$preosteoclasts, can secrete platelet-derived growth factor-BB (PDGF-BB), which is essential for angiogenesis with coupled osteogenesis to maintain bone homeostasis in healthy mice (46). PDGF-B is a ligand of platelet-derived growth factor receptor- $\beta$ (PDGFR- $\beta$ ). The binding of PDGF-B to PDGFR- $\beta$ activates PDGF-BB/PDGFR- $\beta$ signaling (47), which is critical for vasculogenesis and/or angiogenesis $(48,49)$. In addition, autocrine or paracrine activation of this signaling is implicated in a range of conditions, such as cancer and tissue fibrosis $(50,51)$.

In this study, we tested the role of preosteoclast-derived PDGF-BB in the development of the aberrant subchondral bone angiogenesis during osteoarthritis progression. Using destabilization of the medial meniscus (DMM) osteoarthritis mouse models, we found that mononuclear preosteoclasts in subchondral bone/bone marrow of osteoarthritic joints are stimulated very early in mice after DMM surgery and produce a markedly high amount of PDGF-BB, which activates PDGFR- $\beta$ signaling to stimulate aberrant development of subchondral bone angiogenesis with coupled osteogenesis as well as nerve ingrowth. We further generated conditional Pdgfb deletion and transgenic mice, in which PDGF-BB is deleted and overexpressed, respectively, in Trap ${ }^{+}$preosteoclasts, and demonstrated that preosteoclast-derived PDGF-BB is both sufficient to cause and required for aberrant subchondral bone angiogenesis and the resultant joint structural damage and osteoarthritis pain.

\section{Results}

Aberrant subchondral bone angiogenesis develops at preosteoarthritis and early-stage osteoarthritis. To examine the change in subchondral bone blood vessels during osteoarthritis progression, we induced posttraumatic osteoarthritis by performing DMM surgery in C57BL/ 6 mice. Mild proteoglycan loss in cartilage was observed at 4 weeks after surgery and became severe at 6 weeks (Figure 1A). Osteoarthritis Research Society International (OARSI) score was increased at 4 and 6 weeks after surgery, with the increase at 6 weeks being more profound (Figure 1B). Neither obvious proteoglycan loss in AC nor increased OARSI score was detected in the joints of mice at 2 weeks after surgery compared with the sham-operated mice (controls). Consistently, 3-dimensional microcomputed tomography $(\mu \mathrm{CT})$ analysis showed that the increase in tibial subchondral bone volume/total volume (BV/TV) started at 4 weeks and was further aggravated at 6 weeks after surgery (Figure 1, C and D). The thickness of subchondral bone plate (SBP Th) (Figure 1E) and trabecular pattern factor (Tb Pf) (Figure $1 \mathrm{~F}$ ) were also increased at 4 and 6 weeks after surgery in DMM mice compared with controls, indicating uneven bone formation. These subchondral bone parameters were unchanged at 2 weeks postoperatively in DMM mice relative to controls. We then detected type $\mathrm{H}$ vessels $\left(\mathrm{CD} 31^{\mathrm{hi}}\right.$ endomucin $\left.{ }^{\mathrm{hi}}\left[\mathrm{Emcn}^{\mathrm{hi}}\right]\right)$, which have been recognized as osteogenesis-coupling neo-vessels responsible for new bone formation $(46,52,53)$, in subchondral bone of DMM mice. An increase in $\mathrm{CD} 31^{\text {hi }} \mathrm{Emcn}^{\text {hi }}$ blood vessels in subchondral bone/bone marrow was found as early as 2 weeks and was sustained until 6 weeks after DMM surgery, whereas the neo-vessel formation in AC was detected at 6 weeks after DMM surgery (Figure 1, G and H). Of note, neo-vessels were also found in joint cartilage in the DMM mice (Figure 1G, bottom right), suggesting the invasion of new vessels into the calcified cartilage during the progression of OA. Thus, the development of aberrant subchondral bone angiogenesis starts at pre- and early-stage osteoarthritis development, preceding joint structure damage. 
A

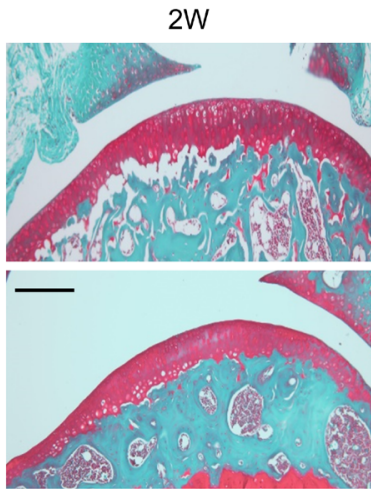

C

Sham

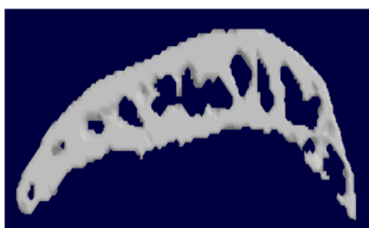

DMM
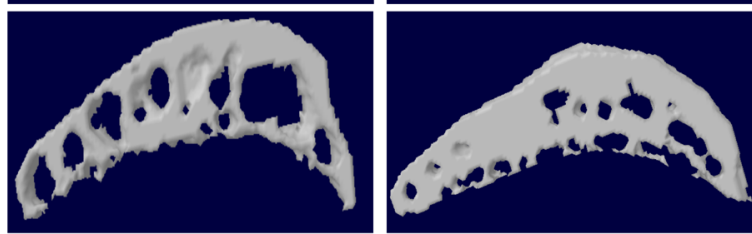

G

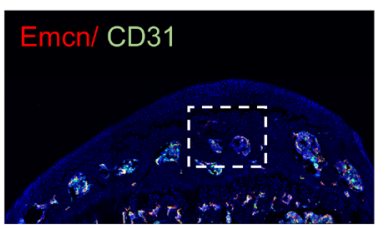

Sham
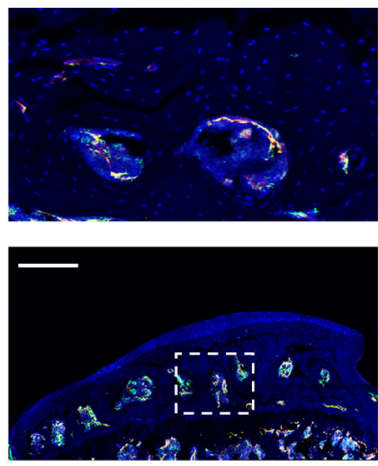

DMM

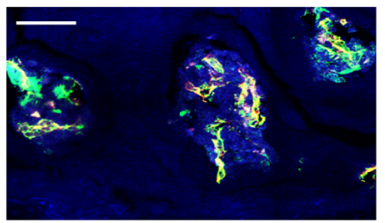

$4 \mathrm{~W}$
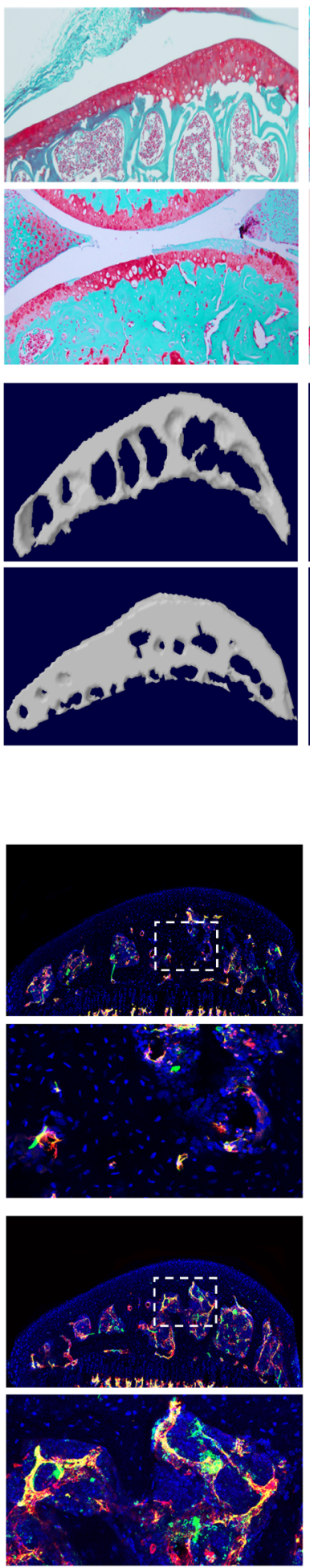

$6 \mathrm{~W}$
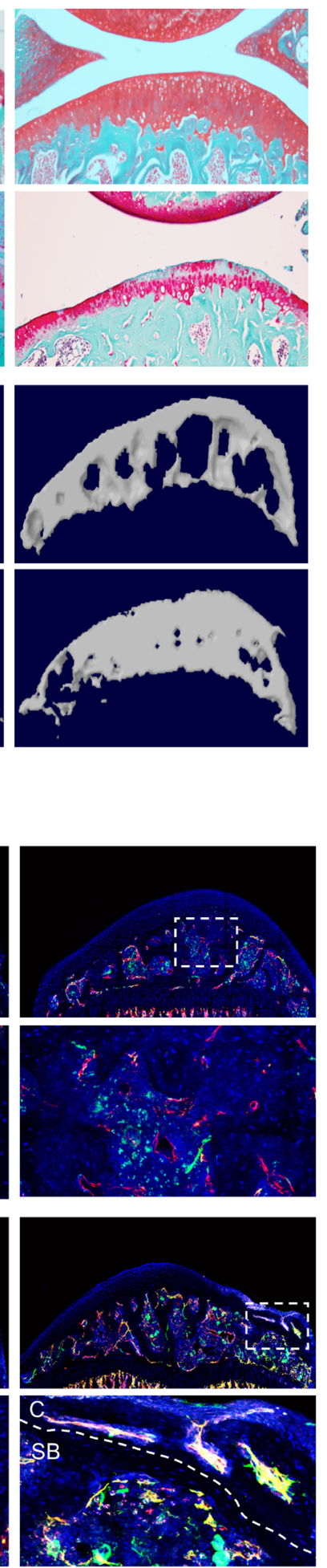
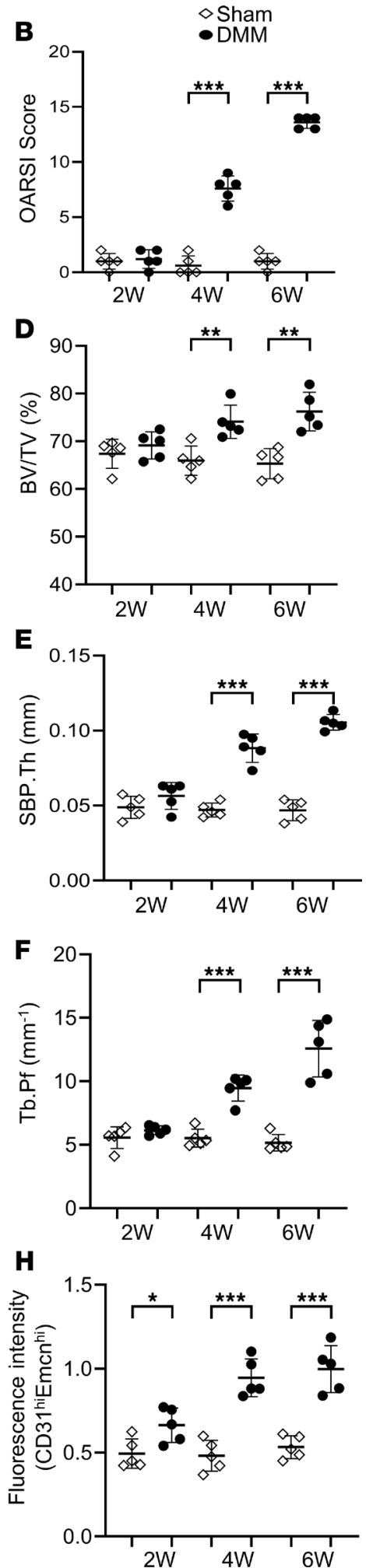

Figure 1. Aberrant subchondral bone angiogenesis develops at preosteoarthritis and early-stage osteoarthritis. Three-month-old C57BL/ 6 mice underwent destabilization of the medial meniscus (DMM) or sham surgery. Knee joints were harvested at 2, 4, and 6 weeks after surgery. $n=5$ mice per group. (A) Safranin 0-fast green staining of the tibia subchondral bone medial compartment (sagittal view). Scale bar: $200 \mu \mathrm{m}$. (B) Calculation of Osteoarthritis Research Society International (OARSI) scores. ${ }^{* *} P<0.001$. (C-F) Three-dimensional microcomputed tomography ( $\mu$ CT) images (C) and quantitative analysis of structural parameters of subchondral bone: bone volume/tissue volume (BV/TV) (D), subchondral bone plate thickness (SBP. Th, mm) (E), and trabecular pattern factor (Tb. Pf, $\left.\mathrm{mm}^{-1}\right)(\mathbf{F}) .{ }^{* *} P<0.01$, and ${ }^{* * *} P<0.001$. (G and $\left.\mathbf{H}\right)$ Immunofluorescence staining of CD31 (green) and endomucin (Emcn) (red) with quantification of the intensity of $C D 31^{\text {hi }} \mathrm{Emcn}^{\text {hi }}$ signal per tissue area in subchondral bone of the tibia. Scale bars: $200 \mu \mathrm{m}$ (top), $40 \mu \mathrm{m}$ (bottom). ${ }^{*} P<0.05$, and ${ }^{* *} P<0.001$. C, cartilage; SB, subchondral bone. All data are shown as means \pm standard deviations. Statistical significance was determined by unpaired, 2-tailed Student's $t$ test. 
Preosteoclasts secrete an excessive amount of PDGF-BB, which activates PDGFR- $\beta$ signaling in pericytes to promote angiogenesis in osteoarthritic subchondral bone. We previously reported that bone/bone marrow mononuclear preosteoclasts secrete PDGF-BB, which is a critical bone angiogenesis factor in healthy mice (46). We examined whether PDGF-BB mediates the development of aberrant subchondral bone angiogenesis during osteoarthritis progression. Immunofluorescence staining showed increased PDGF-BB-expressing cells in subchondral bone/bone marrow of DMM mice relative to controls (Figure 2, A and B). Approximately $93.81 \% \pm 5.72 \%$ and $93.14 \% \pm 4.82 \%$ of the cells expressing PDGF-BB were F $4 / 80^{+}$(Supplemental Figure 1, A and B; supplemental material available online with this article; https://doi.org/10.1172/jci. insight.135446DS1) and RANK ${ }^{+}$osteoclast precursors (Supplemental Figure 1, D and E). The data are consistent with our previous finding that PDGF-BB is almost exclusively expressed in mononuclear preosteoclasts (46). Moreover, the percentages of $\mathrm{F} 4 / 80^{+}$and $\mathrm{RANK}^{+}$cells that express PDGF-BB were both significantly increased in subchondral bone of DMM mice relative to sham control mice (Supplemental Figure 1, C and F). Consistently, ELISA analysis revealed much higher levels of PDGF-BB in subchondral bone/bone marrow of DMM mice compared with controls at 2 and 4 weeks after surgery (Figure 2C), indicating that elevation of PDGF-BB in subchondral bone is an early event. Of note, serum PDGF-BB concentration was also markedly higher in DMM mice at 2 weeks after surgery relative to controls (Figure 2D). In addition, phosphorylated PDGFR- $\beta^{+}$(p-PDGFR- $\beta^{+}$) cells were increased in subchondral bone/ bone marrow in DMM mice versus controls (Figure 2, E and F), as detected by immunofluorescence staining. Importantly, triple-immunofluorescence staining revealed that $\mathrm{p}-\mathrm{PDGFR}-\beta^{+}$cells almost exclusively covered the neo-vessels that were $\mathrm{CD} 31^{\text {hi }}$ and $\mathrm{Emcn}^{\text {hi }}$ (Figure 2, E and G), indicating the activation of PDGF-B/PDGFR- $\beta$ signaling in pericytes that were recruited for neo-vessel formation. These data show that in response to joint injury, preosteoclasts produce excessive PDGF-BB, which activates PDGFR- $\beta$ signaling in pericytes to stimulate angiogenesis in subchondral bone/bone marrow.

Deletion of PDGF-BB in preosteoclasts attenuates aberrant subchondral bone angiogenesis in osteoarthritic joints. To investigate whether PDGF-BB is required for the development of aberrant subchondral bone angiogenesis during osteoarthritis progression, we used Trap ${ }^{+}$lineage-specific conditional Pdgfb deletion mice (Pdgfb ${ }^{\mathrm{cKO}}$ ) by crossing Trap-Cre mice with Pdgfb-floxed mice. Because the Trap-Cre line was previously found to have germline transmission, we conducted a characterization of this Trap-Cre strain using TRAP-Cre Rosa26-tdTomato mice, in which the $\mathrm{TRAP}^{+}$cells and their descendants are permanently labeled by tdTomato fluorescence. In addition to bone tissue, we did find tdTomato ${ }^{+}$cells in other tissues, such as brain and aorta (Supplemental Figure 2A). However, while the majority of the tdTomato ${ }^{+}$cells (close to 80\%) in subchondral bone expressed PDGF-BB, we did not detect any PDGF-BB expression in brain and aorta (Supplemental Figure 2, A and B). We further examined whether PDGF-BB is specifically expressed in preosteoclasts in subchondral bone/bone marrow using the conditional Pdgfb deletion mice. While almost all the $\mathrm{RANK}^{+}$cells in subchondral bone/bone marrow expressed PDGF-BB in the Pdgfb-floxed (WT) mice, $\mathrm{PDGF}-\mathrm{BB}^{+}$cells were almost undetectable in the $\mathrm{RANK}^{+}$cells in $\mathrm{Pdgfb}^{\mathrm{cKO}}$ mice (Figure 3, A and B), indicating an effective deletion of PDGF-BB in preosteoclasts in subchondral bone. Together, these results demonstrate that although there is a nonspecific Cre expression in the cell types other than osteoclast lineage and in nonbone tissues, PDGF-BB is exclusively expressed in bone preosteoclasts. Therefore, the off-target deletion of Pdgfb by using TRAP-Cre can be excluded.

Consistent with the immunofluorescence staining result in Figure 3, A and B, we detected much lower PDGF-BB concentration in subchondral bone/bone marrow of Pdgfb ${ }^{c K O}$ mice relative to WT mice (Figure 3C). Importantly, abundant type $\mathrm{H}$ vessels and osteoprogenitor osterix ${ }^{+}$cell clusters were formed in the subchondral bone/bone marrow of the WT mice after DMM surgery, whereas Pdgfb ${ }^{\text {cKO }}$ mice had markedly reduced angiogenesis (Figure 3, D and E) and osteogenesis in subchondral bone (Figure 3, F and G). We postulated that increased subchondral bone innervation may also occur in osteoarthritis mice because the neo-vessels often promote the growth of nerve fibers. Indeed, abundant nerve fibers were detected in subchondral bone in WT mice after DMM surgery and were reduced in the Pdgfb ${ }^{\mathrm{cKO}}$ mice (Figure $3 \mathrm{H}$, upper, and Figure 3I). Moreover, consistent with the neo-vessel invasion into the cartilage in osteoarthritis mice in Figure $1 \mathrm{H}$, nerve fibers were also found in joint cartilage in the WT mice after DMM surgery (Figure 3H, bottom left panel), indicating the nerve ingrowth into the calcified cartilage during the progression of osteoarthritis (OA). The invasion of the nerves in cartilage was not found in the $\mathrm{Pdgfb}{ }^{\mathrm{cKO}}$ mice (Figure $3 \mathrm{H}$, bottom right).

Conditional PDGF-BB-knockout mice are protected from joint damage. We examined microarchitectural changes in the subchondral bone of the Pdgfb ${ }^{\text {cKO }}$ mice. Tibia subchondral BV/TV, SBP Th, and Tb Pf 
A

Sham

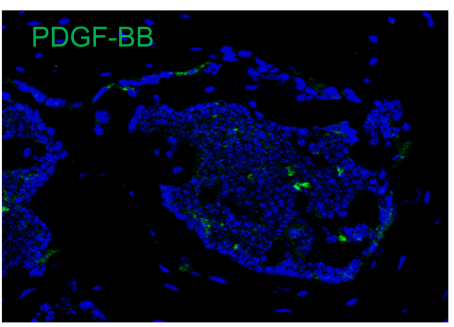

C

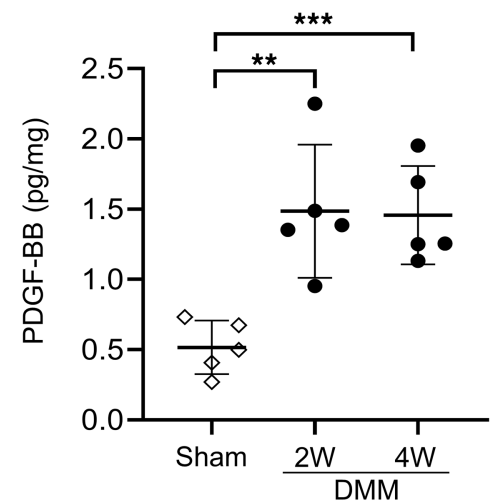

DMM

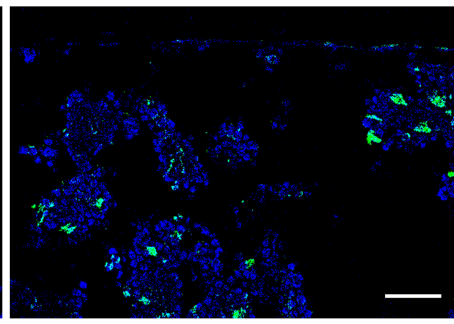

D

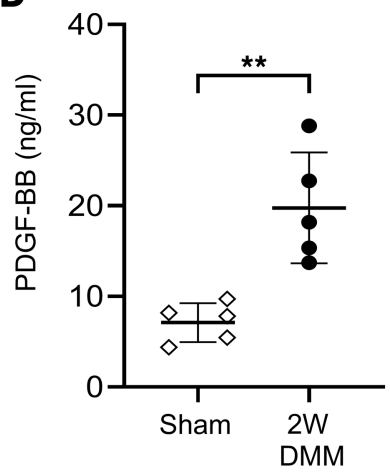

B

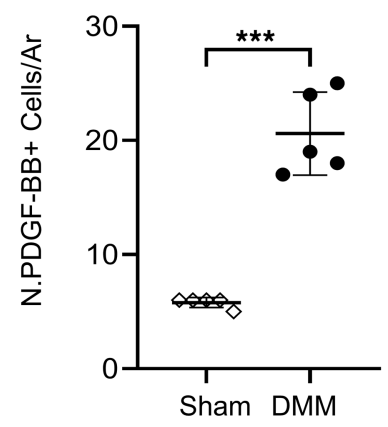

E
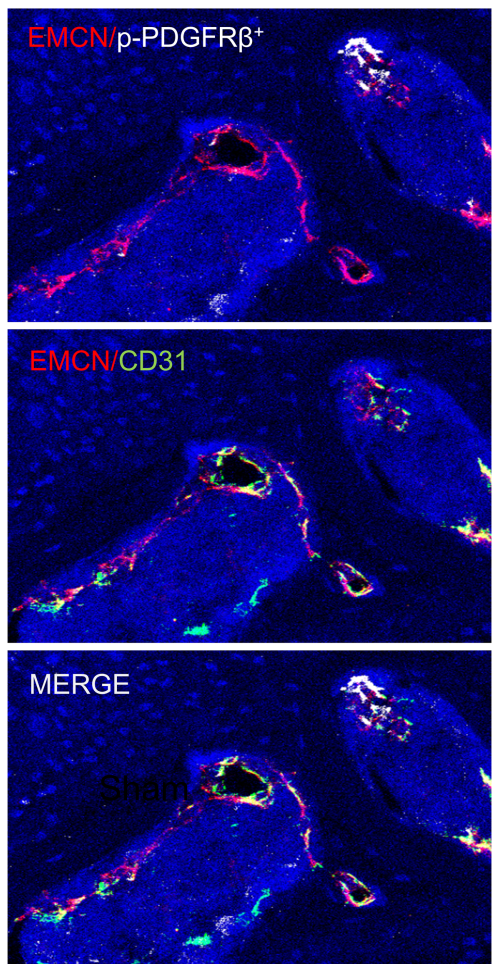

DMM
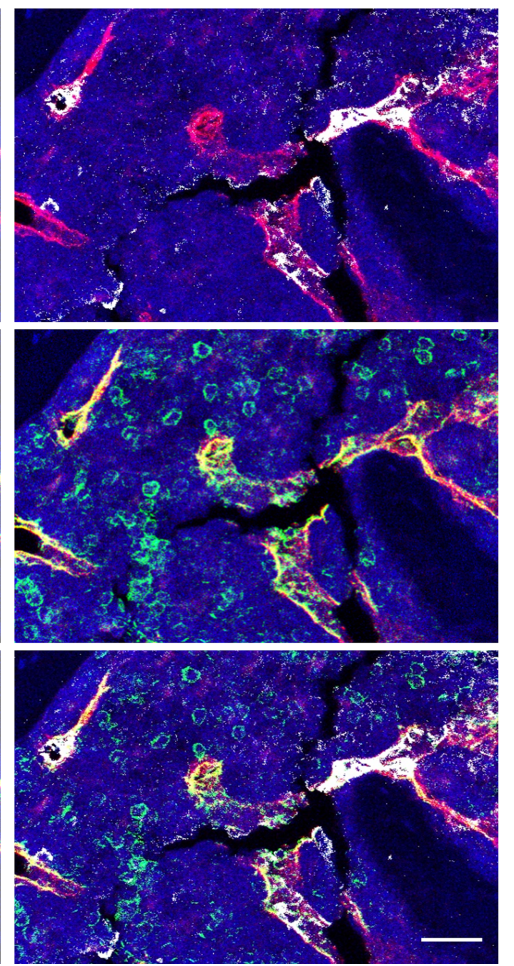

F
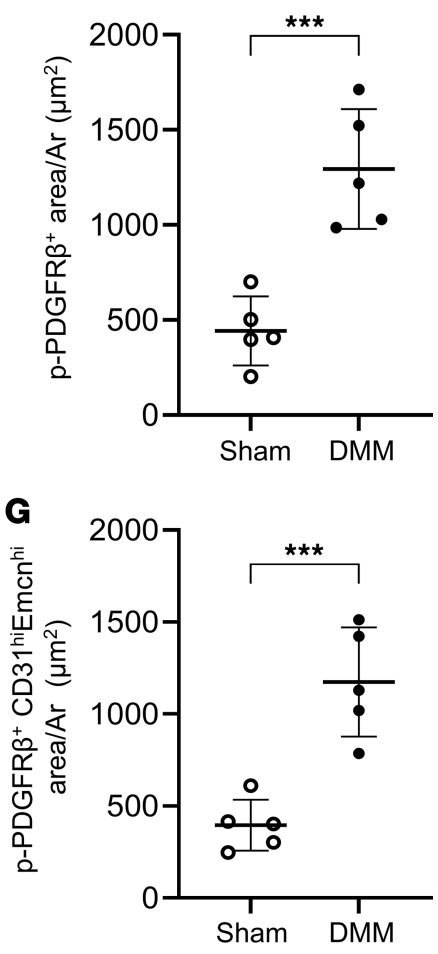

Figure 2. Preosteoclasts secrete an excessive amount of PDGF-BB, which activates PDGFR- $\beta$ signaling in subchondral bone blood vessel cells. Threemonth-old C57BL/6 mice underwent DMM or sham surgery. Knee joints were harvested at 2 weeks after surgery. $n=5$ mice per group. (A and B) Immunostaining of PDGF-BB (green) with quantification of PDGF-BB ${ }^{+}$cells per tissue area in subchondral bone of the tibia. Scale bar: $50 \mu \mathrm{m} .{ }^{* * *} P<0.001$. Ar, total view area. (C) ELISA analysis of PDGF-BB protein concentration in tibial subchondral bone/bone marrow in mice at 2 weeks and 4 weeks after DMM surgery. ${ }^{* *} P<0.01$, and ${ }^{* *} P<0.001$. (D) ELISA analysis of PDGF-BB protein concentration in serum in mice at 2 weeks after DMM surgery. ${ }^{* *} P<$ 0.01. (E-G) Triple-immunofluorescence staining of p-PDGFR- $\beta$ (white), CD31 (green), and endomucin (red) (E). The areas of $p-P D C F R-\beta^{+}$(F) and p-PDGFR- $\beta^{+}$CD $31^{\text {hi }} E m \mathrm{En}^{\text {hi }}$ (G) signals $/ \mu \mathrm{m}^{2}$ view field were calculated using Imagel $(\mathrm{NIH})$. Scale bar: $50 \mathrm{~mm}$. ${ }^{* * *} P<0.001$. All data are shown as means \pm standard deviations. Statistical significance was determined by unpaired, 2-tailed Student's $t$ test. area/Ar, area/total view area. 
A

DMM

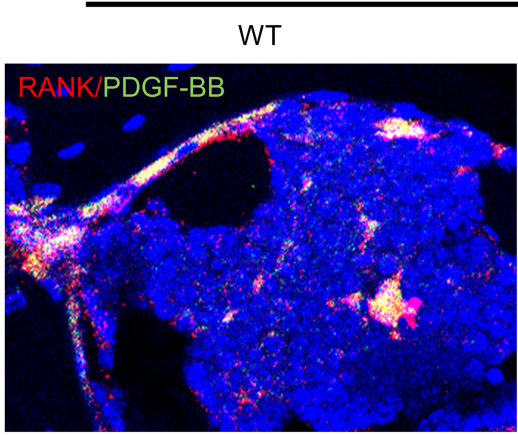

D
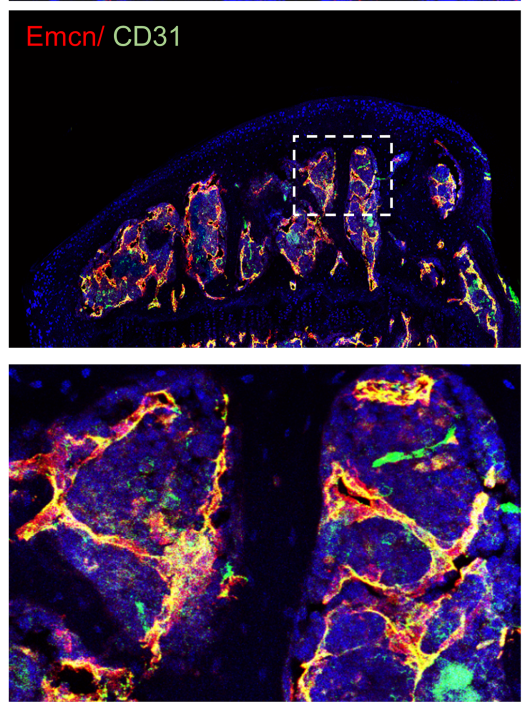

$\mathbf{F}$

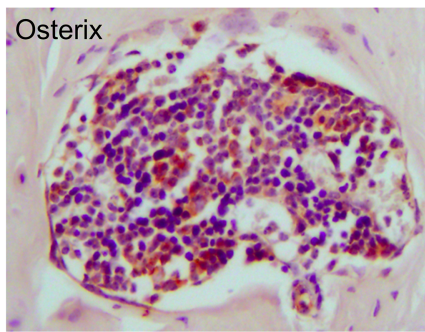

H
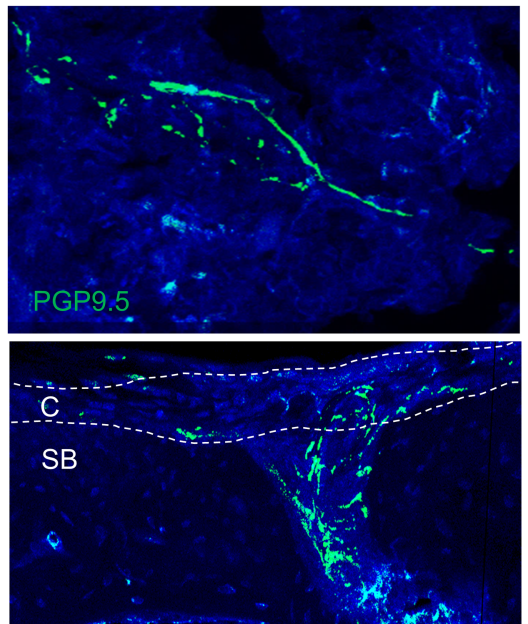

Pdgfbcko
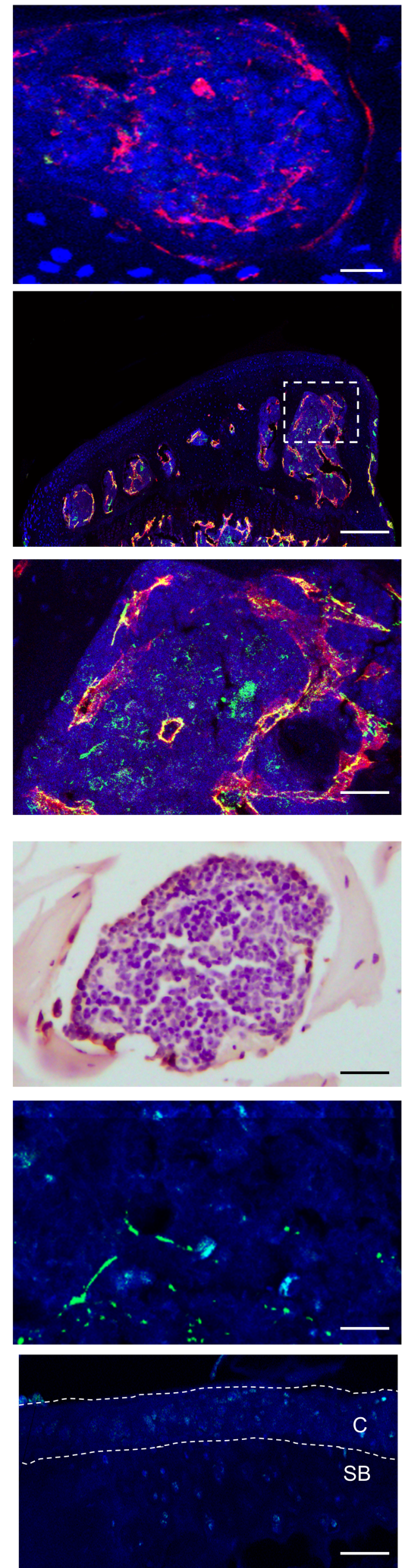

$\diamond$ WT

B - Pdgfb ${ }^{c K O}$

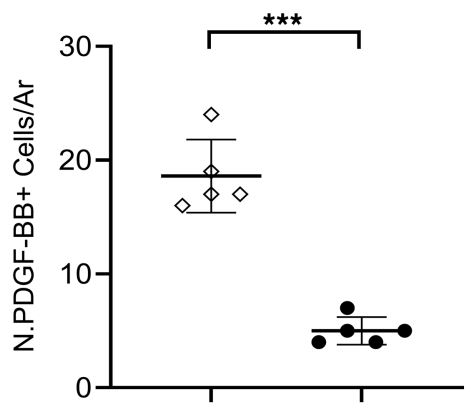

C

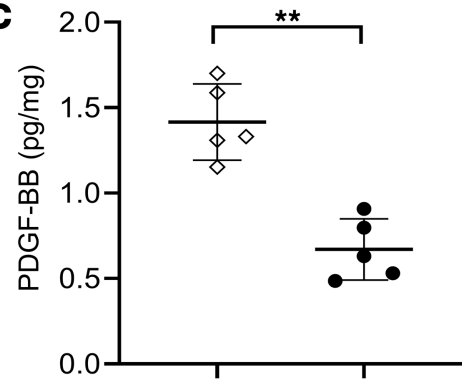

E

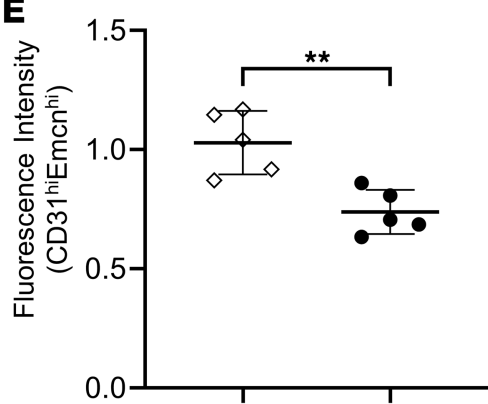

G

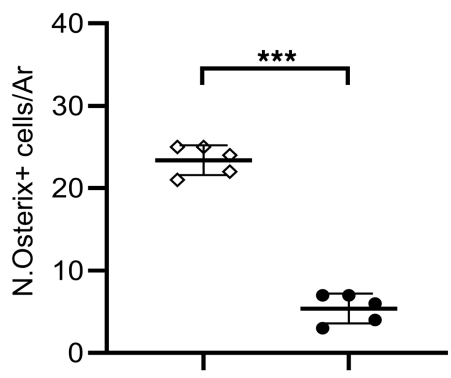

I

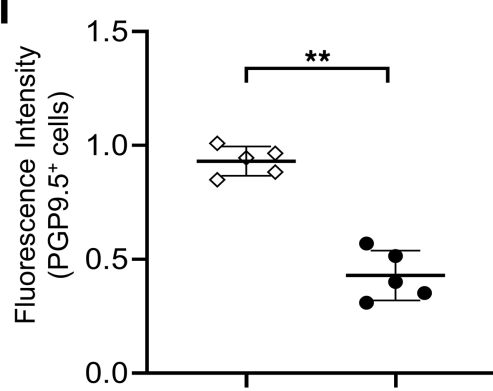


Figure 3. Deletion of PDGF-BB in preosteoclasts attenuates aberrant joint subchondral bone angiogenesis. Three-month-old Trap-Cre Pdgfb $\mathrm{fl}^{\mathrm{f} / \mathrm{f}} \mathrm{mice}$

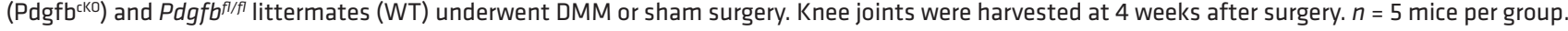
(A and B) Immunostaining of RANK (red) and PDGF-BB (green) and the quantification of PDGF-BB+ cells per tissue area in tibial subchondral bone. Scale bar: $50 \mu \mathrm{m} .{ }^{* *} P<0.001$. (C) ELISA analysis of PDGF-BB concentration in tibial subchondral bone/bone marrow. ${ }^{* *} P<0.01$. (D and E) Immunofluorescence staining of CD31 (green) and Emcn (red) with quantification of the intensity of CD31 ${ }^{\text {hi } E m c n ~ h i ~ s i g n a l ~ p e r ~ t i s s u e ~ a r e a ~ i n ~ s u b c h o n d r a l ~ b o n e ~ o f ~ t h e ~}$ tibia. Scale bars: $200 \mu \mathrm{m}$ (top), $50 \mu \mathrm{m}$ (bottom). ${ }^{* *} P<0.01$. (F and $\mathbf{G}$ ) Immunohistochemical analysis of Osterix (brown) and quantification of Osterix cells in tibial subchondral bone. Scale bar: $50 \mu \mathrm{m}$. ${ }^{* *} P<0.001$. (H and I) Immunofluorescence staining of PGP9.5 (green) in joints. Upper panel images show PGP9. $5^{+}$nerves in subchondral bone only (scale bar: $50 \mu \mathrm{m}$ ), and bottom panel images show PGP9. $5^{+}$nerves in both joint cartilage and subchondral bone (scale bar: $40 \mu \mathrm{m}$ ). C, cartilage; SB, subchondral bone. (I) Quantification of the intensity of PGP9.5 signal per tissue area in subchondral bone of the tibia. ${ }^{*} P<0.01$. All data are shown as means \pm standard deviations. Statistical significance was determined by unpaired, 2 -tailed Student's $t$ test.

were all increased in the WT mice $\left(\mathrm{Pdgfb}^{+/+}\right)$after DMM relative to controls. All of these parameters were almost normalized in the Pdgfb ${ }^{\mathrm{cKO}}$ mice to the levels of the controls (Figure 4, A-D). We also evaluated the cartilage phenotype of the mice by histological analysis. Proteoglycan loss and calcification of AC were significantly lower in Pdgfb ${ }^{\mathrm{cKO}}$ mice than in WT mice after DMM (Figure 4E). The protective effects on $\mathrm{AC}$ in $\mathrm{Pdgfb}{ }^{\mathrm{cKO}}$ mice were also reflected in OARSI scores (Figure 4F). Moreover, after DMM surgery, WT mice exhibited loss of spontaneous activities, which were more or less improved in the Pdgfb ${ }^{\text {cKO }}$ mice (Figure 4, G-J). von Frey test showed that WT mice after DMM surgery relative to sham surgery exhibited mechanical hyperalgesia of the hind paw, as indicated by the increased paw withdrawal frequency and decreased 50\% paw withdrawal threshold (Figure 4, K-M). Paw withdrawal frequency was reduced (Figure $4, \mathrm{~K}$ and $\mathrm{L}$ ), but the improvement of the $50 \%$ paw withdrawal threshold was not significant (Figure $4 \mathrm{M}$ ) in the Pdgfb ${ }^{\mathrm{cKO}}$ mice (vs. WT mice) after DMM surgery, indicating that joint hyperalgesia to pressure stimuli may not be significantly relieved in the Pdgfb ${ }^{\text {cKO }}$ mice. CatWalk analysis revealed a significant difference between the ratio of left/right hind paw ipsilateral intensity (Figure $4 \mathrm{~N}$ ) and contact area (Figure 4O) in WT mice after DMM surgery relative to sham surgery, and this difference was significantly reduced in the $\mathrm{Pdgfb}^{\mathrm{cKO}}$ mice.

Transgenic mice expressing PDGF-BB in preosteoclasts recapitulate the subchondral bone changes of osteoarthritic joints. To examine whether preosteoclast-produced excessive PDGF-BB is sufficient to induce subchondral bone angiogenesis, we generated conditional transgenic mice, Pdgfb ${ }^{\text {cTG }}$ mice, in which PDGF-BB is expressed in the $\mathrm{TRAP}^{+}$cells by ligation of a $2.8-\mathrm{kb}$ full-length human $P D G F B$ gene with a Trap ${ }^{+}$cell-specific promoter tartrate-resistant acid phosphatase 5 (TRACP5) (Figure 5A). Three transgenic founder lines were produced. One of the transgenic founder lines was established for further study. Immunofluorescence staining of subchondral bone/bone marrow tissue sections showed that the number of $\mathrm{PDGF}-\mathrm{BB}^{+}$cells was markedly elevated in the mice relative to WT mice (Figure 5, B and C). The level of PDGF-BB in the subchondral bone/bone marrow was doubled in Pdgfb ${ }^{\text {cTG }}$ mice (vs. WT mice) in ELISA analysis (Figure 5D). We then assessed the changes of joint subchondral bone in these mice. Intriguingly, many more CD31 ${ }^{\text {hi }} \mathrm{Emcn}^{\text {hi }}$ blood vessels were detected in the subchondral bone/bone marrow in 5-month-old Pdgfb ${ }^{\text {сTG }}$ mice compared with the WT mice (Figure 5, E and F). Moreover, neo-vessels were also found in the joint cartilage in $\mathrm{Pdgfb}^{\mathrm{cTG}}$ mice (Figure $5 \mathrm{E}$ ). Osterix ${ }^{+}$osteoprogenitor cells (Figure 5, G and $\mathrm{H}$ ) and PGP9. $5^{+}$nerve fibers (Figure 5, I and J) were also increased the subchondral bone marrow of Pdgfb ${ }^{\mathrm{cTG}}$ mice. Increases in the tibia subchondral BV/TV (Figure 5, K and L), SBP Th (Figure 5M), and Tb Pf (Figure 5N) were detected in Pdgfb ${ }^{\mathrm{cTG}}$ mice relative to WT mice. Therefore, transgenic mice exhibited aberrant subchondral bone angiogenesis with a progressive invasion of new vessels into the joint calcified cartilage as well as an increase in subchondral bone osteogenesis and innervation, accurately mirroring the subchondral bone changes of osteoarthritic joints.

Transgenic mice expressing PDGF-BB in preosteoclasts develop osteoarthritis spontaneously. We then examined the changes in joint cartilage of the Pdgfb ${ }^{\mathrm{TTG}}$ mice. Pdgfb ${ }^{\mathrm{TTG}}$ at 5 months of age exhibited severe proteoglycan loss and apparent damage of cartilage tissue (Figure 6A). The OARSI scores of Pdgfb ${ }^{\text {TTG }}$ mice were much higher compared with the age-matched WT mice (Figure 6B). To examine specific changes in extracellular matrix, we analyzed expression of collagen type $\mathrm{X} \alpha 1$ chain (Co110A1) and matrix metallopeptidase 13 (Mmp13) in AC tissue from Pdgfb ${ }^{\text {TTG }}$ mice and WT mice. Immunostaining data showed abundant staining for Col10A1 (Figure 6, C and D) and Mmp13 (Figure 6, E and F) in the cartilage of Pdgfb ${ }^{\text {cTG }}$ mice, compared with minimal expression in WT mice.

Finally, we assessed functional changes in the Pdgfb ${ }^{\text {cTG }}$ mice by performing pain behavior tests. We first assessed spontaneous activity, which indicates the potential effects of pain. Significant differences in distance 
A

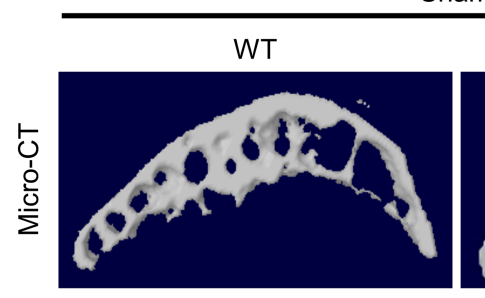

Sham

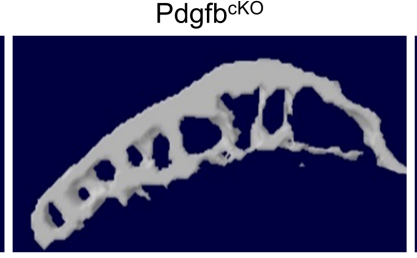

DMM

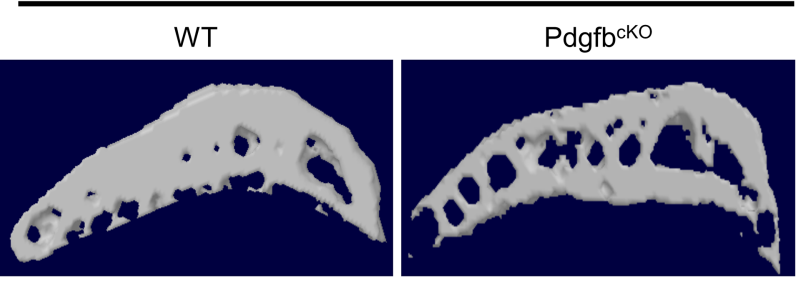

$\mathbf{E}$
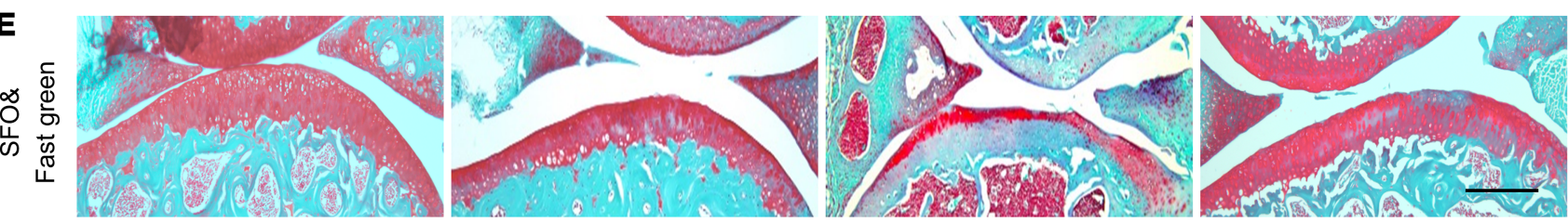

$\triangle$ WT-Sham

$\Delta$ PdgfbcKo - Sham

○ WT-DMM

- PdgfbcKo $-D M M$
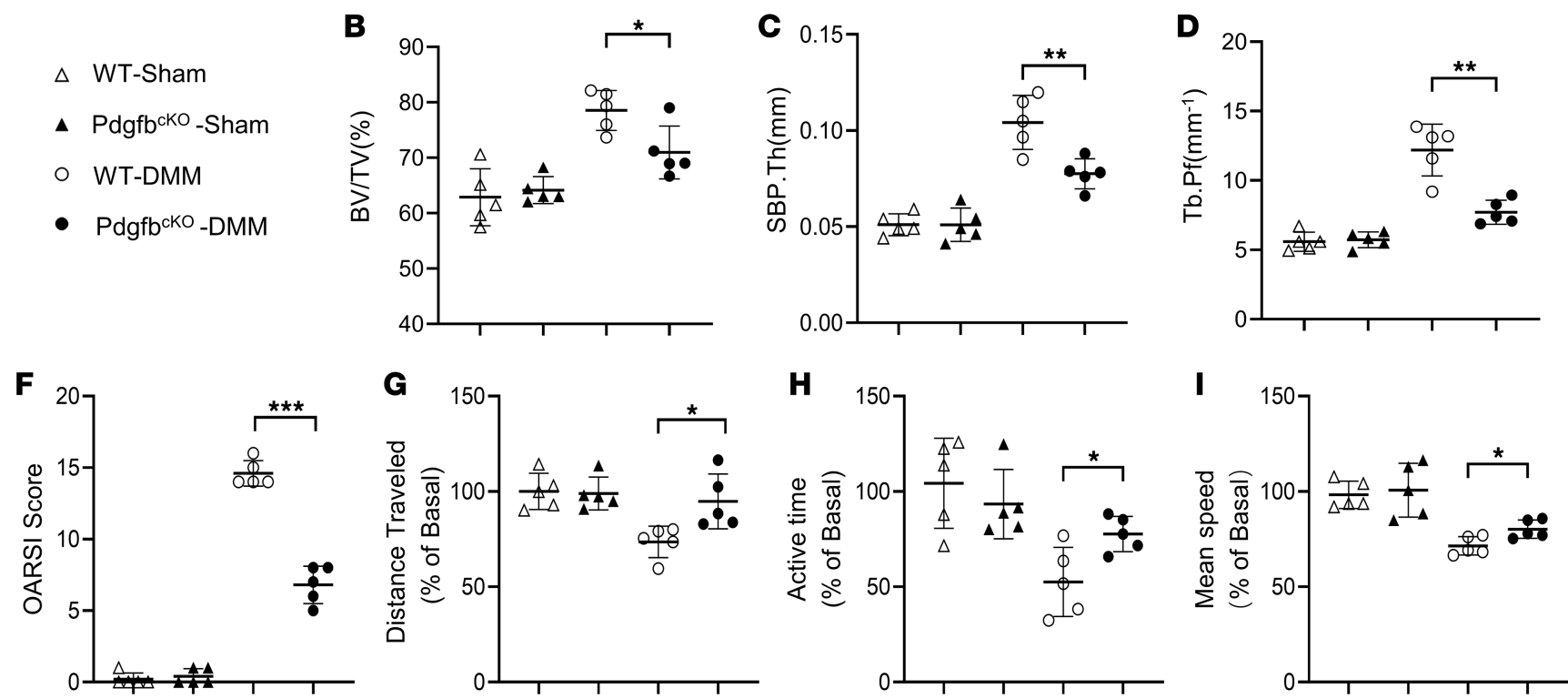

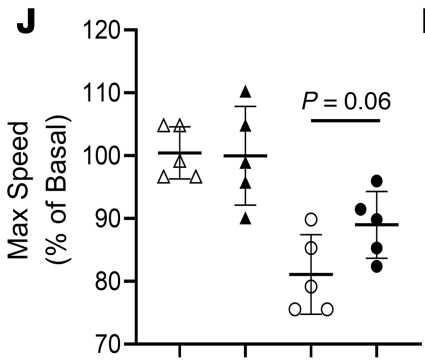

$\mathbf{K}$
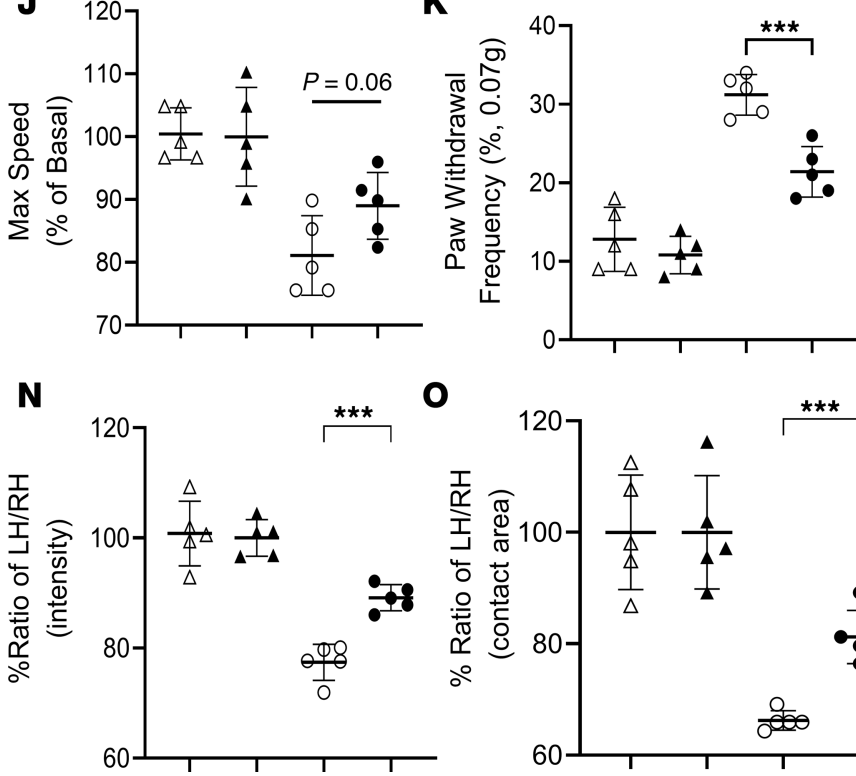

o

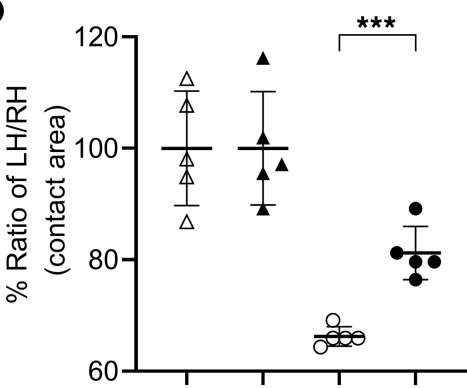

$\mathbf{L}$

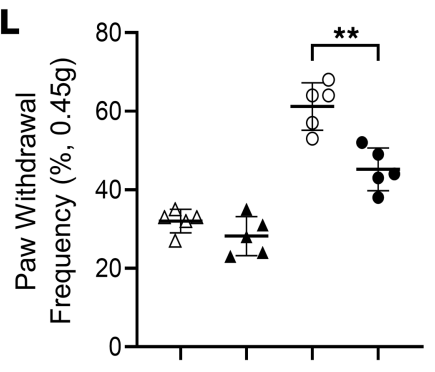

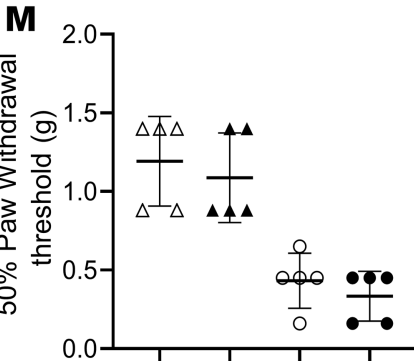

Figure 4. Conditional PDGF-BB-knockout mice are protected from joint damage. Three-month-old Trap-cre Pdgfb ${ }^{f / / 7}$ mice $\left(P d g f b^{c K O}\right)$ and Pdgfb ${ }^{f / f l}$ littermates (WT) underwent DMM or sham surgery. Knee joints were harvested at 6 weeks after surgery. $n=5$ mice per group. (A-D) Three-dimensional $\mu \mathrm{CT}$ images and quantitative analysis of structural parameters of subchondral bone: BV/TV, SBP Th (mm), and Tb. Pf $\left(\mathrm{mm}^{-1}\right)$. ${ }^{*} P<0.05$, and ${ }^{* *} P<0.01$. (E) Safranin 0 -fast green staining of tibial subchondral bone medial compartment (sagittal view). Scale bar: 200 $\mu \mathrm{m}$. (F) Calculation of OARSI scores. ${ }^{* *} P<0.001$. (G-J) Voluntary wheel running measurements: distance $(\mathbf{C})$, active time $(\mathbf{H})$, mean speed $(\mathbf{I})$, and maximum speed $(\mathrm{J})$. ${ }^{*} P<0.05$ determined by the percentage of sham surgery mice. (K-M) Paw withdrawal threshold measurement. ${ }^{* *} P<0.01$, and ${ }^{* *} P<0.001$. ( $\left.\mathbf{N}-\mathbf{0}\right)$ Ratio (left hind/right hind paws) of intensity $(\mathbf{N})$ and contact area $(\mathbf{0})$ shown based on CatWalk analysis. ${ }^{* * *} P<0.001$. All data are shown as means \pm standard deviations. $P$ value was calculated by 2-way ANOVA. 
A

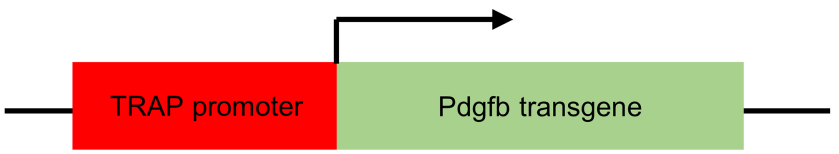

B

WT

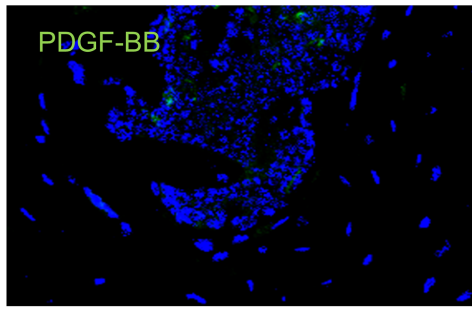

$\mathbf{E}$
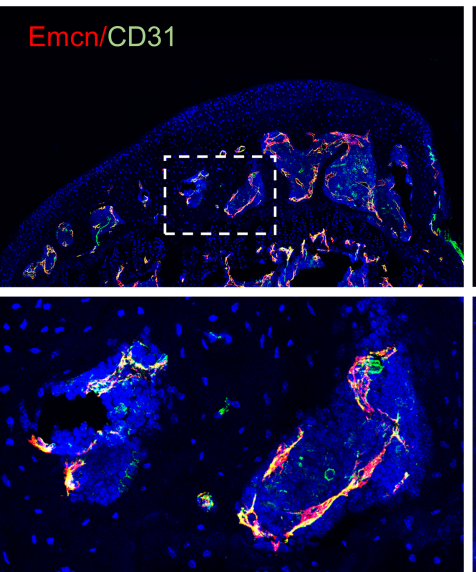

G

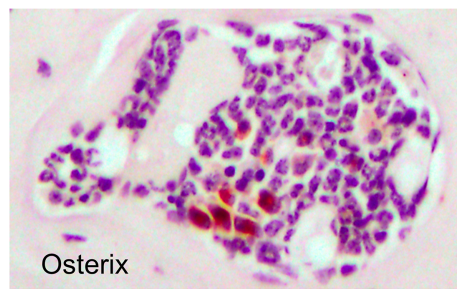

I

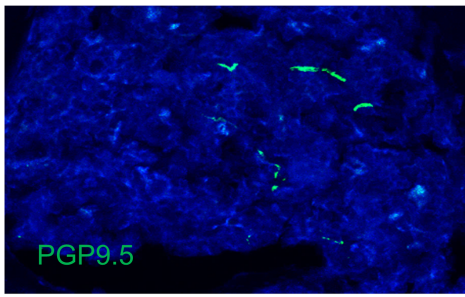

$\mathbf{K}$
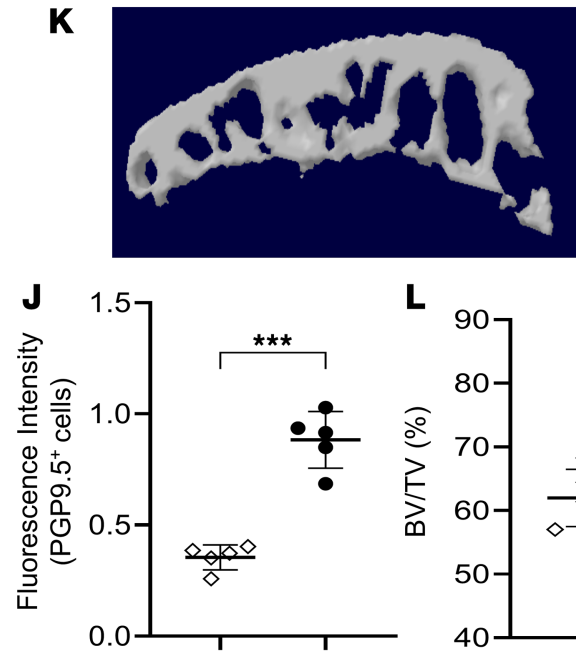

PdgfbcTG
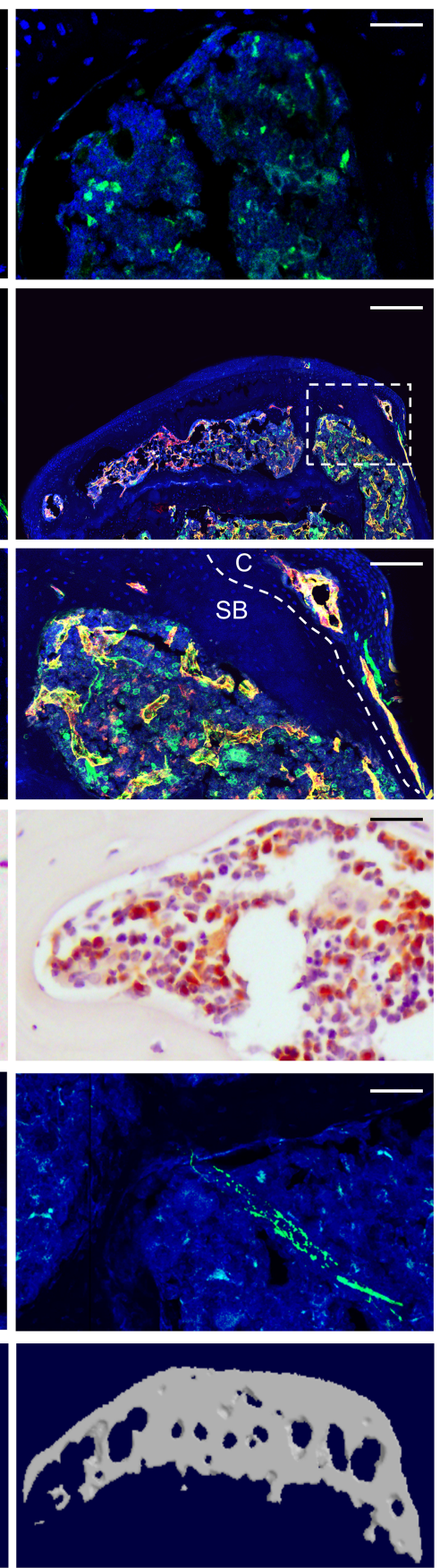

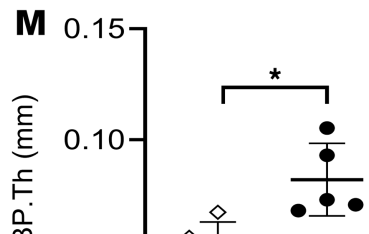

क $0.05-\stackrel{8}{\stackrel{0}{8}}$ $\diamond$ WT

- PdgfbcTG
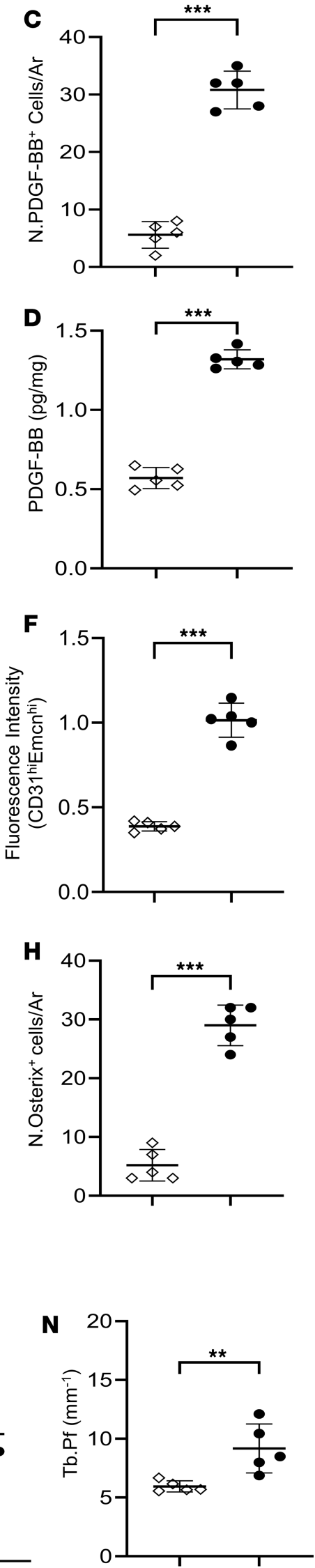
Figure 5. Transgenic mice expressing PDGF-BB in preosteoclasts recapitulate the pathological features of osteoarthritic joint subchondral bone. (A) Schematic diagram showing the TRACP5-Pdgfb transgene in the transgenic mice (Pdgfb ${ }^{\mathrm{cTC}}$ ). (B-N) Knee joints were harvested from 5-month-old transgenic mice and WT mice. $n=5$ mice per group. Immunofluorescence staining of PDCF-BB (green) and quantification of PDGF-BB ${ }^{+}$cells in tibial subchondral bone (B and $\mathbf{C}$ ). Scale bar: $50 \mu \mathrm{m}$. ${ }^{* *} P<0.001$. ELISA analysis of PDGF-BB concentration in tibial subchondral bone/bone marrow.

${ }^{* *} P<0.001$ (D). Immunofluorescence staining of CD31 (green) and Emcn (red) with quantification of the intensity of CD31hiEmcn ${ }^{\text {hi }}$ signal per tissue area in subchondral bone of the tibia (E and F). C, cartilage; SB, subchondral bone. Scale bars: $200 \mu \mathrm{m}$ (top), $50 \mu \mathrm{m}$ (bottom). ${ }^{* * *} P<0.001$. Immunohistochemical analysis of Osterix (brown) and quantification of Osterix cells in tibial subchondral bone (G and $\mathbf{H})$. Scale bar: $50 \mu \mathrm{m} .^{* * *} P<0.001$. Immunofluorescence staining of PGP9.5 (green) with quantification of the intensity of PGP9.5 signal per tissue area in subchondral bone of the tibia (I and J). Scale bar: $50 \mu \mathrm{m} .{ }^{* *} P<0.001$. Three-dimensional $\mu \mathrm{CT}$ images (K) and quantitative analysis of structural parameters of subchondral bone: BV/TV (L), SBP. Th $\left(\mathrm{mm}^{-1}\right)(\mathbf{M})$, and Tb. Pf $\left(\mathrm{mm}^{-1}\right)(\mathbf{N}) .{ }^{*} P<0.05$, and ${ }^{* *} P<0.01$. All data are shown as means \pm standard deviations. Statistical significance was determined by unpaired, 2-tailed Student's $t$ test.

traveled, active time, and mean and maximum speed of movement (per 24 hours) were detected between Pdgf$\mathrm{b}^{\mathrm{cTG}}$ mice and WT mice (Figure 6, G-J). von Frey analysis showed that the paw withdraw frequency increased significantly in Pdgfb ${ }^{\mathrm{cTG}}$ mice compared with WT mice of the same age (Figure $6, \mathrm{~K}$ and L). The increase in $50 \%$ paw withdrawal threshold, however, was not statistically significant in Pdgfb ${ }^{\text {cTG }}$ mice (vs. WT mice) (Figure $6 \mathrm{M}$ ). The histology and behavior assessment results suggest that preosteoclast-produced excessive PDGF$\mathrm{BB}$ causes joint degeneration and aggravates structural and functional impairment of osteoarthritic joints.

\section{Discussion}

Angiogenesis, the generation of new blood vessels from preexisting vessels, within an osteoarthritic joint is known to contribute to OA progression $(29,54)$. Particularly, aberrant subchondral bone angiogenesis with resultant invasion of vasculature into the osteochondral junction is a hallmark of human osteoarthritis (55). By using an osteoarthritis rabbit model, Saito et al. showed that angiogenic activity of subchondral bone peaked during the early to progressive stage and decreased to a normal level during the late stage of OA (33), whereas the vascular invasion into AC occurred during the progressive stage after the increase of subchondral angiogenic activity. Consistent with this observation, here we demonstrate that aberrant subchondral bone angiogenesis occurred during pre- and early-OA stages before the joint degeneration occurs. Angiogenesis in cartilage was observed only at a later stage, when structural damage of AC developed (Figure 7). Our results, in agreement with the findings of several previous studies, suggest that neo-vessel formation in subchondral bone is characterized by the development of osteogenesis-coupling type $\mathrm{H}$ vessels (CD $31^{\mathrm{hi}} \mathrm{Emcn}^{\mathrm{hi}}$ ) $(32,34,35,56)$. The simultaneous increase in osteogenesis and subchondral bone microarchitecture alterations that we observed in osteoarthritic mice further support this assumption. In addition, our data further imply that neo-vessels originating in subchondral bone/bone marrow lead to eventual AC damage and joint pain-associated behavior changes through 2 ways. On one hand, the neo-vessels enable osteoid islet formation in subchondral bone/bone marrow to alter stress distribution on the AC, leading to its degeneration. On the other hand, angiogenesis promotes subchondral bone innervation. The development of the neo-vessels and nerves may be coordinated and gradually invade avascular cartilage together, eventually leading to AC degeneration and joint pain (Figure 7).

Our results pinpoint the crucial role of PDGF-BB in the development of pathological subchondral bone angiogenesis during pre- and early-stage $\mathrm{OA}$ and identify preosteoclasts as a primary source of the excessive PDGF-BB in the bone/bone marrow microenvironment. Our prior work demonstrated that, under normal, healthy conditions, bone/bone marrow $\mathrm{TRAP}^{+}$preosteoclasts secrete $\mathrm{PDGF}-\mathrm{BB}$, which is required for the maintenance of bone homeostasis (46). In this study, we revealed that, after traumatic joint injury, mononuclear preosteoclasts secreted excessive amounts of PDGF-BB, which activates PDGFR- $\beta$ signaling in bone/bone marrow vascular cells and pericytes in a paracrine manner for aberrant neo-vessel formation (Figure 7). Our data from conditional Pdgfb-knockout mice further show that preosteoclast-derived PDGF$\mathrm{BB}$ is required for pathological subchondral bone angiogenesis and resultant joint degeneration. Therefore, it is important that PDGF-BB concentration in the bone/bone marrow microenvironment be maintained within a physiological range. PDGF-BB deficiency causes bone loss (46), whereas too much PDGF-BB production by preosteoclasts may lead to the development of osteoarthritis. Notably, the PDGF-BB concentrations in both subchondral bone and serum are markedly higher in mice at 2 weeks after DMM surgery relative to sham operation, suggesting that PDGF-BB may serve as an early diagnostic biomarker of OA. It would be interesting to conduct a human population-based study in the future to further validate the result from animals. The finding that conditional $P d g f b$-transgenic mice accurately recapitulate the subchondral 
A
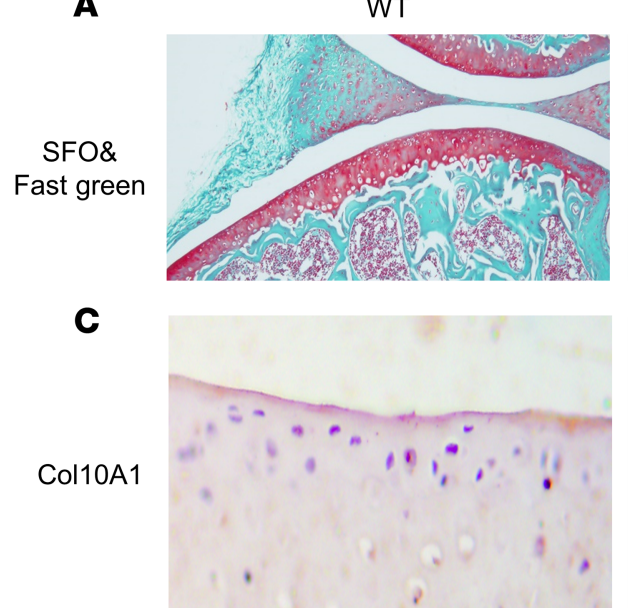

E

MMP13

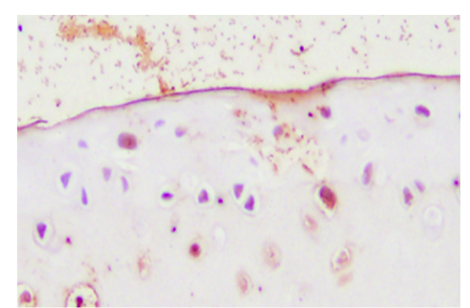

Pdgfb ${ }^{\text {TG }}$
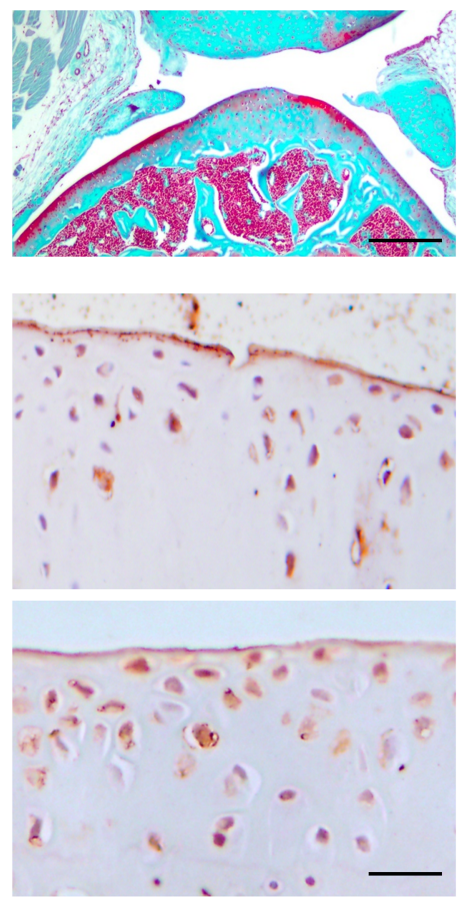

B $\quad \stackrel{W T}{\text { Pdgfb } \mathrm{cTG}}$
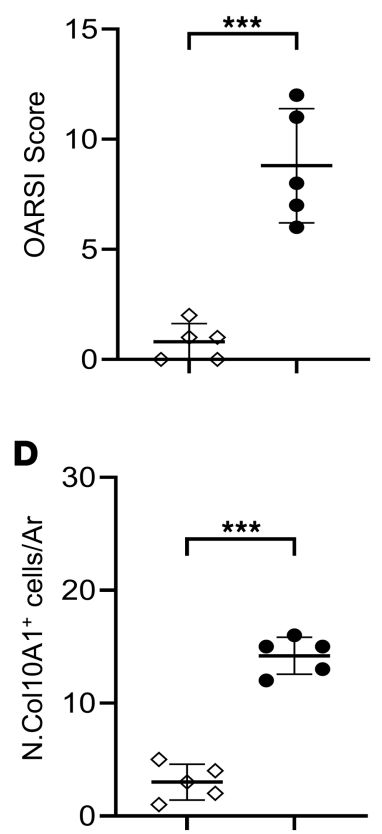
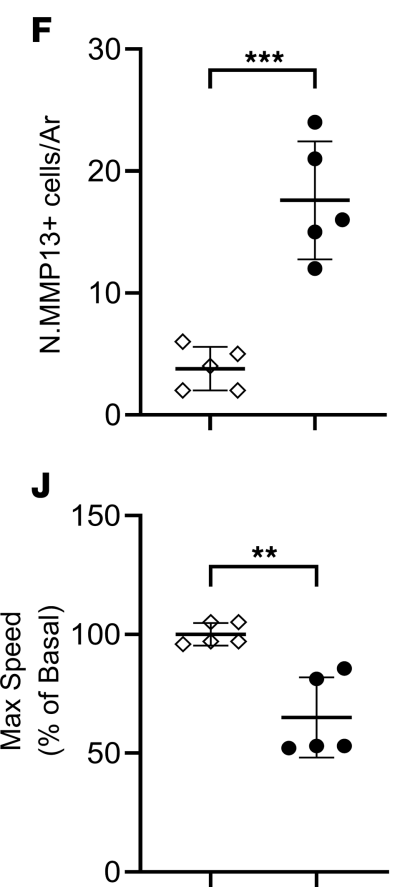

G

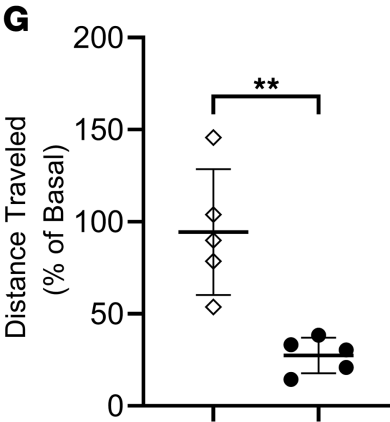

$\mathbf{K}$

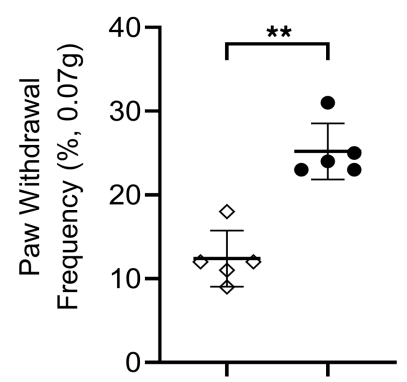

H
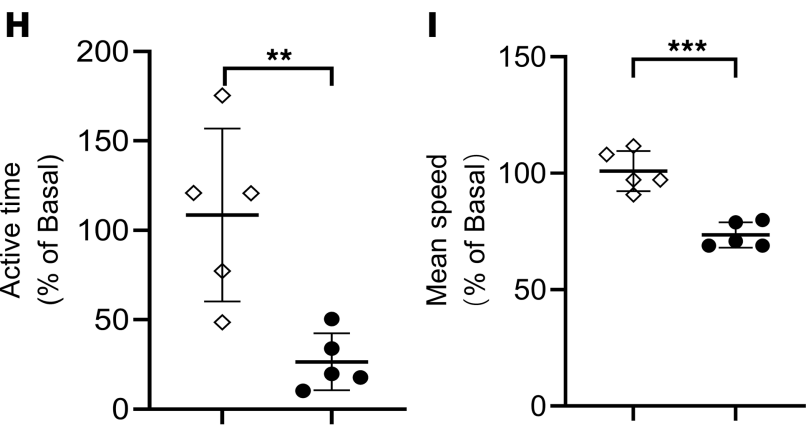

$\mathbf{L}$

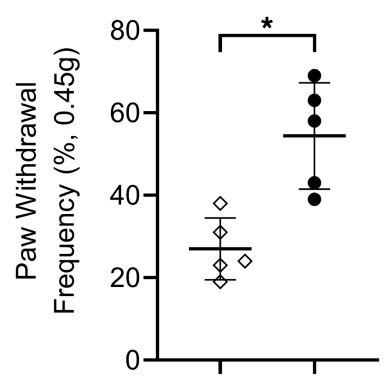

M

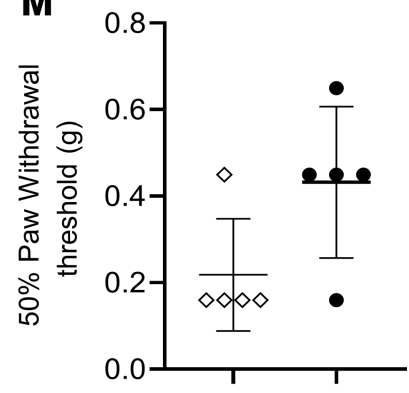

Figure 6. Transgenic mice expressing PDGF-BB in preosteoclasts develop OA spontaneously. Knee joints were harvested from 5-month-old transgenic mice (Pdgfb ${ }^{c T C}$ ) and WT mice. $n=5$ mice per group. (A) Safranin 0 -fast green staining of tibial subchondral bone medial compartment (sagittal view). Scale bar: $200 \mu \mathrm{m}$. (B) Calculation of the OARSI scores. ${ }^{* *} P<0.001$. (C-F) Immunohistochemical staining of collagen type $X \alpha 1$ chain (Col10A1) (C) and matrix metallopeptidase 13 (Mmp13) (brown) (E) and quantification of Col10A1 ${ }^{+}$(D) and Mmp13+ cells (F) in tibial subchondral bone. Scale bar: $100 \mu \mathrm{m} .{ }^{* * *} P<0.001$. (G-J) Voluntary wheel running measurements: distance (G), active time (H), mean speed (I), and maximum speed (J). ${ }^{* *} P$ $<0.01$, and ${ }^{* *} P<0.001$ determined by the percentage of sham surgery mice. (K-M) Paw withdrawal threshold measurement. ${ }^{*} P<0.05$, and ${ }^{* *} P<$ 0.01 determined by the percentage of sham surgery mice. All data are shown as means \pm standard deviations. Statistical significance was determined by unpaired, 2-tailed Student's $t$ test. 


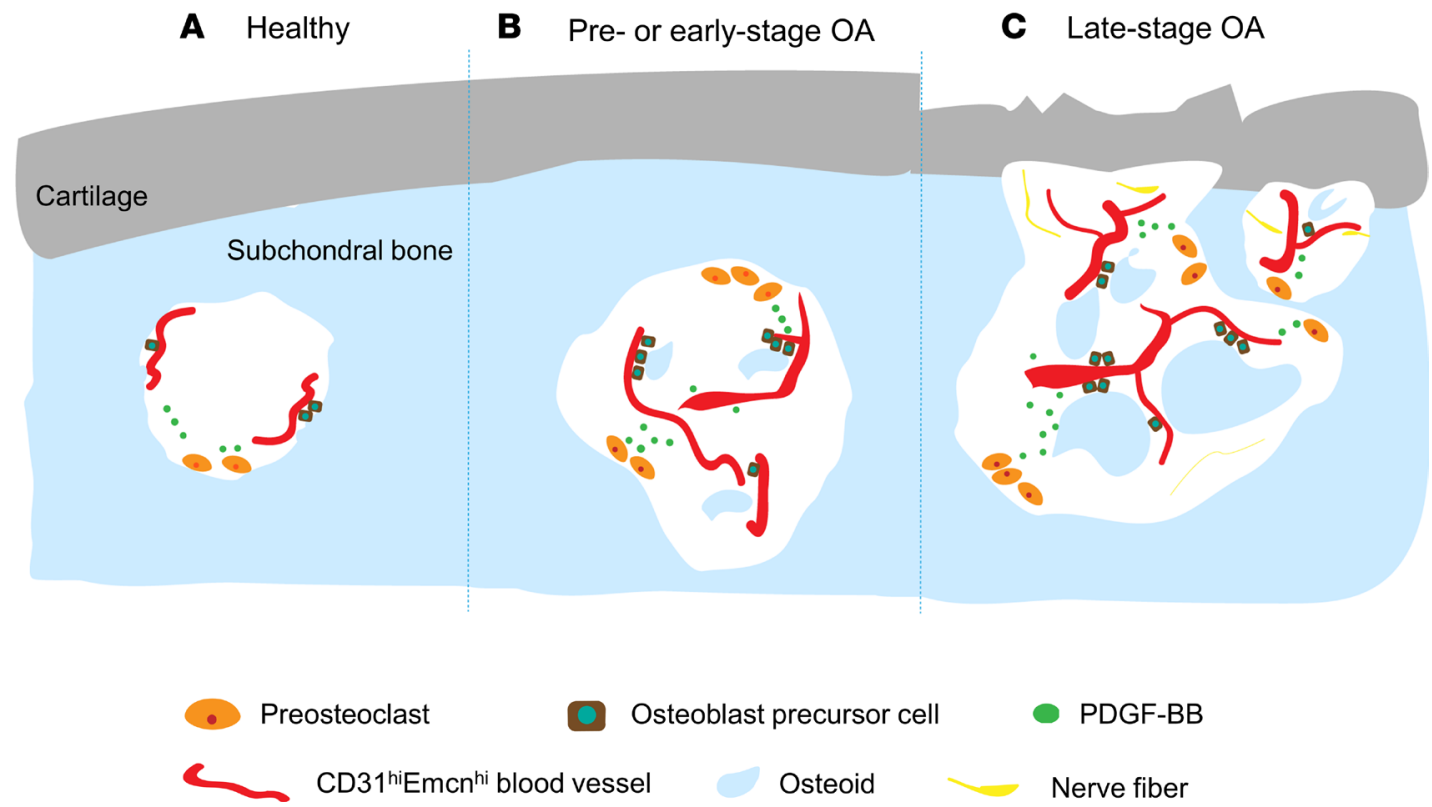

Figure 7. Schematic model of the involvement of preosteoclast-derived PDGF-BB in the development of OA. (A) In uninjured, healthy joints, PDCF-BB level in subchondral bone/bone marrow microenvironment is within a normal range, and neo-vessels develop only at the bone surface where new bone formation occurs. (B) After joint injury or under disease conditions, mononuclear preosteoclasts are activated and secrete excessive amounts of PDCF-BB, which triggers aberrant angiogenesis coupled with osteogenesis and innervation in subchondral bone/bone marrow. (C) The neo-vessels and nerves gradually invade avascular cartilage to induce $A C$ ossification and promote bone marrow osteoid islet formation, and both alterations together lead to joint degeneration and $\mathrm{OA}$ pain.

bone angiogenesis phenotype and develop osteoarthritis spontaneously at a young age further indicates that preosteoclast-derived increases in PDGF-BB are an initial driving force for OA progression. This transgenic mouse model thus provides a valuable tool to study the pathophysiological mechanisms underlying OA progression and to develop treatment for this most common joint disorder.

Other mechanisms may contribute to subchondral bone angiogenesis during OA development. Although the involvement of vascular endothelial growth factor (VEGF) and the signaling pathway in the angiogenesis of AC and synovium (55) in late-stage OA have been well studied, there is limited research on the mechanisms underlying subchondral bone angiogenesis at the initial stage in OA progression. A previous study reported that leucine rich alpha-2-glycoprotein 1 (LRG1), which regulates epithelial-mesenchymal transition and angiogenesis in colorectal cancer, may regulate pathogenic subchondral bone angiogenesis because increased LRG1 was found in the subchondral bone and AC in anterior cruciate ligament transection (ACLT) mice (57). The increased LRG1 in subchondral bone was detected at 30 days after ACLT surgery, when AC degeneration had already occurred (16, 34), suggesting that LRG1 may regulate subchondral bone angiogenesis at a relatively late stage of posttraumatic osteoarthritis. A recent study by Lu et al., using the DMM OA mouse model, demonstrated that activated mTOR complex 1 in the hypertrophic chondrocytes in AC mediated the production of VEGF from the chondrocytes, resulting in subchondral bone angiogenesis at 5 weeks after DMM surgery (32). During new vessel growth and remodeling, the concentration and activity of angiogenesis factors in the local environment must be controlled and coordinated precisely to induce formation and stabilization of new vessels (58). In addition to recruiting pericytes to stabilize blood vessels, PDGF-BB can directly induce endothelial cell proliferation, migration, and tube formation, as well as stimulate VEGF secretion (59). Thus, it is possible that PDGF-BB acts in concert with other proangiogenic factors, such as VEGF, to induce neo-vessel formation in subchondral bone in osteoarthritic joints. Nevertheless, our finding that deletion of PDGF-BB in preosteoclasts almost abolished pathological subchondral bone angiogenesis and joint damage strongly implies a crucial role of PDGF-BB in the development of aberrant subchondral bone angiogenesis during pre- and early-stage OA.

Why preosteoclasts secrete more PDGF-BB after joint injury is an interesting question. In addition to bone-resorptive activity, osteoclasts are known to regulate neighboring cells through secretion of an array of factors, known as "clastokines" (42). However, because PDGF-BB is secreted primarily by mononuclear 
preosteoclasts but not by multinuclear, mature osteoclasts (46), the number and/or activity of preosteoclasts in subchondral bone/bone marrow may be increased under uneven mechanical loading after joint injury, leading to excessive secretion of PDGF-BB. Indeed, we observed an increase in the number of preosteoclasts in subchondral bone/bone marrow after DMM surgery. Future work is required to determine whether increased PDGF-BB production by preosteoclasts is at the transcriptional or the posttranslational level and how the process is initiated during OA development. In addition to mechanistic insights into subchondral bone angiogenesis and its role in OA pathogenesis as presented in the current study, the profound joint structural and functional improvements in the conditional Pdgfb-knockout mice suggest that targeting preosteoclasts or PDGF-BB/PDGFR- $\beta$ signaling in subchondral bone may provide a promising approach for the prevention and early treatment of OA. We are aware that the pathogenic mechanisms of posttraumatic and naturally occurring OA could be different, which is an interesting topic for our future study. Future study is needed to determine the role of PDGF-BB in the disease development of other OA subtypes, such as spontaneous aging OA and metabolic dysregulation-associated OA.

Our data also reveal an association of PDGF-BB with subchondral bone innervation during OA development. We found that PDGF-BB deletion in preosteoclasts almost abrogated the aberrant nerve growth in subchondral bone of the DMM mice. Conversely, aberrant nerve growth in subchondral bone developed spontaneously in the conditional $P d g f b$-transgenic mice. We detected type $\mathrm{H}$ neo-vessels and nerve fibers in joint cartilage in DMM mice, indicating a coinvasion of the blood vessels and nerves into the calcified cartilage during the progression of $\mathrm{OA}$, which may lead to AC degeneration and joint pain. Indeed, the results from the pain-associated behavior tests, especially the CatWalk test and the spontaneous activity tests, confirmed that osteoarthritis pain behavior was exacerbated by overexpression of $P d g f b$ and reduced by knockout of $P d g f b$ in preosteoclasts. It remains unclear whether the nerve growth PDGF-BB induces is a direct effect or indirect by promoting other nerve growth factors' production in a paracrine fashion. We also note that subchondral and cartilage innervation PDGF-BB induces may not be the only contributor to OA pain, and our finding does not exclude the possible involvement of synovial hyperplasia/inflammation and synovial innervation in this process. Nevertheless, given that subchondral bone angiogenesis also promotes sensory nerve ingrowth along the newly formed blood vessels $(54,60,61)$, targeting preosteoclasts or PDGF-BB may have the potential to prevent and/or treat osteoarthritis pain. The current work provides proof-of-concept evidence for the role of preosteoclast-derived PDGF-BB in the development of OA. Future human population-based study is needed to further validate these findings.

\section{Methods}

Mouse generation. Pdgffl/fl mice were purchased from The Jackson Laboratory. The Trap-Cre mouse strain was obtained from Jolene J. Windle (Virginia Commonwealth University, Richmond, Virginia, USA). We crossed Trap-Cre mice with Pdgff $b^{f / f l}$ mice (mice homozygous for the Pdgfb-floxed allele are referred to as "Pdgfb ${ }^{+/+} "$ in the text) to generate Trap-Cre Pdgffl/fl mice (referred to as "PdgfbcKO" in the text). We determined the genotype of the mice by PCR analyses of genomic DNA isolated from mouse tails using the same primers described previously (46).

The mouse TRACP5 promoter was ligated with 2.8-kb full-length human PDGFB cDNA and subcloned into a pBluescript plasmid. Transgenic mice were produced by pronuclear injection of C57BL/6 fertilized eggs at the Transgenic Mouse Core Facility (Johns Hopkins University, School of Medicine). Primers used for genotyping the transgenic mice were as follows: mouse TRACP5 forward: TTAACTCCTGGGACTCTGAA; human Pdgfb reverse 1: AGTGGTCACTCAGCATCTCAT; human Pdgfb reverse 2: GCTCAGCAATGGTCAGGGAA; and human Pdgfb reverse 3: ACACCAGGAAGTTGGCGTTG. Unique product lengths of 1000,900 , and $800 \mathrm{bp}$ were generated. All animals were housed in our institution's animal facility.

DMM OA mouse model. DMM surgery was performed on the left knee joints of mice, as previously described (62). Briefly, male C57BL/6 mice were assigned to DMM or sham groups, anesthetized (with ketamine $80-100 \mathrm{mg} / \mathrm{kg}$, xylazine $4-6 \mathrm{mg} / \mathrm{kg}$, and acepromazine $1-2 \mathrm{mg} / \mathrm{kg}$ ), and subjected to medial arthrotomy of the left knee. In the DMM group, the medial meniscotibial ligament of the left joint was exposed and transected with microiris scissors. Controls underwent medial arthrotomy of the left knee without severing the medial meniscotibial ligament. After surgery, mice were monitored daily for the first week and 3 times per week during the study for signs of physical distress.

$\mu C T$ analysis. $\mu \mathrm{CT}$ analysis of the tibial subchondral bone was performed as previously described (16) with modification. The knee joint was dissected, fixed overnight in $4 \%$ formaldehyde, and analyzed by $\mu \mathrm{CT}$ (Skyscan 1174, Bruker MicroCT) (voltage, $65 \mathrm{kVp}$; current, $153 \mu \mathrm{A}$; resolution, $9 \mu \mathrm{m} /$ pixel). Image reconstruction 
software (NRecon v1.6, Bruker), data analysis software (CTAn v1.9, Bruker), and 3-dimensional model visualization software ( $\mu \mathrm{CTVol}$ v2.0, Bruker) were used to analyze the parameters of the tibia subchondral bone. Three-dimensional histomorphometric analysis was performed on cross-sectional images of the tibia subchondral bone. We defined the region of interest as the whole subchondral bone medial compartment, and we used 10 consecutive images from the medial tibial plateau for 3-dimensional reconstruction and analysis. Three-dimensional structural parameters analyzed were BV/TV, Tb Pf, and SBP Th.

Immunocytochemistry, immunofluorescence, and histomorphometry. Mouse knee joints were harvested after euthanasia. For sections, the bones were fixed in $4 \%$ formaldehyde overnight, decalcified in 1.5 M EDTA $(\mathrm{pH}=7.4)$ for 14 days (frozen sections) or 21 days (paraffin sections), and embedded in OCT or paraffin. Immunostaining was performed using standard protocol. For immunofluorescence staining, we incubated the sections with RANK (R\&D Systems, Bio-Techne, 1:100, AF692), F4/80 (Abcam, 1:100, ab100790), PGP9.5 (1:100, ab10404, Abcam), CD31 (Abcam, 1:50, polyclonal), endomucin (Santa Cruz, 1:50, V.7C7), and PDGF-BB (Abcam, 1:50, polyclonal) antibodies followed by fluorescence-linked secondary antibodies (Abcam, ab150120, ab150115, ab150077, ab150078, ab150160). For immunocytochemistry staining, we incubated the sections with Osterix (Abcam, 1:50, V.7C7), Col10A1 (Abcam, ab58632, 1:200), and Mmp13 (Abcam, ab39012, 1:100). A horseradish peroxidase-streptavidin detection kit (Dako) was used in immunohistochemical procedures to detect immunoreactivity, followed by counterstaining with hematoxylin (Dako, S3309). Paraffin sections were used for Safranin O-fast green (MilliporeSigma) staining. Fluorescence images were acquired by using the ZEISS LSM 780 Confocal (with Fluorescence Correlation Spectroscopy).

ELISA of PDGF-BB concentration in serum and subchondral bone/bone marrow extracts. The concentration of PDGF-BB in serum and bone/bone marrow protein extracts was determined by using the Mouse/Rat PDGFBB Quantikine ELISA Kit (R\&D Systems, Bio-Techne) according to the manufacturer's instructions. For the preparation of subchondral bone/bone marrow extracts, tibia bones were isolated and cleaned of connective tissue. The tibial subchondral bone cap with bone marrow was then dissociated under a dissecting microscope. The cap was flash-frozen in liquid nitrogen and pulverized in frozen stainless steel pulverizers. The resulting tissue power was transferred to prefrozen Eppendorf tubes with RIPA buffer and placed on ice for 30 minutes followed by 1 hour rotating at $4^{\circ} \mathrm{C}$ (cold room).

Voluntary wheel running measurement and von Frey test. For voluntary wheel running measurement, an open surface of a wheel was placed inside the mouse cage to allow the mice to run freely. Rotations were transmitted electronically to the system (model BIO-ACTIVW-M, Bioseb) (63) to capture running data. Mice were housed individually, a 24-hour measurement was done, and distance traveled (m), active time(s), mean speed $(\mathrm{m} / \mathrm{mm})$, and maximum speed $(\mathrm{m} / \mathrm{mm})$ were recorded.

von Frey testing was performed according to previously published methods (60). Mice were placed in elevated Plexiglas chambers on metal mesh flooring. A von Frey hair (force range $\approx 0.07,0.45 \mathrm{~g}$ ) was used perpendicular to the plantar surface of the hind paw (avoiding the toe pads) until it just bent, then held in place for 2-3 seconds. After the first difference in response was observed, 4 more measurements were made. The $50 \%$ paw withdrawal threshold was determined using the following formula: $10(\mathrm{Xf}+\mathrm{k} \delta) / 10,000$, where $\mathrm{Xf}$ is the value (in log units) of the final von Frey hair used, $\mathrm{k}$ is the tabular value for the pattern of the last 6 positive/negative responses, and $\delta$ is the mean difference (in log units) between stimuli. The threshold force required to elicit withdrawal of the paw (median 50\% withdrawal) was determined twice on each hind paw (and averaged) on each testing day, with sequential measurements separated by at least 5 minutes.

CatWalk analysis. CatWalk gait analysis system (Noldus Information Technology) was used in this study. Each mouse was placed individually in the walkway and allowed to walk freely and traverse from one side to the other of the walkway. When the mouse paws made contact with the glass plate, light was recorded with a high-speed color video camera that was connected to a computer running software. The software automatically labeled all contacted areas and assigned them to the respective paws. The parameters (mean intensity, paw print area) were generated.

Statistics. All data are presented as means \pm standard deviations. For comparisons between 2 groups, we used unpaired, 2-tailed Student's $t$ tests. For more than 2 groups with multiple measurements, we used 2-way ANOVA for those experiments. For all experiments, $P<0.05$ was considered significant $\left({ }^{*} P<0.05\right.$, ${ }^{* *} P<0.01$, and $\left.{ }^{* * *} P<0.001\right)$. All inclusion/exclusion criteria were preestablished, and no samples or animals were excluded from the analysis. No statistical method was used to predetermine the sample size. The experiments were randomized, and the investigators were blinded to allocation during experiments and outcome assessment. The same sample was not measured repeatedly. 
Study approval. The experimental protocol was reviewed and approved by the Institutional Animal Care and Use Committee of The Johns Hopkins University.

\section{Author contributions}

WS and MW designed the experiments; WS carried out most of the experiments; GL, XL, YZ, QS, XW, and $\mathrm{YH}$ helped with some experiments; GZ, PG, SD, and XC proofread the manuscript; and MW supervised the experiments, analyzed results, and wrote the manuscript.

\section{Acknowledgments}

The authors gratefully acknowledge the assistance of Kerry Kennedy and Rachel Box at the Johns Hopkins Department of Orthopaedic Surgery Editorial Services for editing the manuscript. This work was supported by the National Institutes of Health grant R56 AG059578 to MW. We also acknowledge the assistance of Johns Hopkins School of Medicine Microscope Facility supported by the National Center for Research Resources of the National Institutes of Health under award number S10OD016374.

Address correspondence to: Mei Wan, Ross Building, Room 232, 720 Rutland Avenue, Baltimore, Maryland 21205, USA. Phone: 410.502.6598; Email: mwan4@jhmi.edu.

1. Prieto-Alhambra D, Judge A, Javaid MK, Cooper C, Diez-Perez A, Arden NK. Incidence and risk factors for clinically diagnosed knee, hip and hand osteoarthritis: influences of age, gender and osteoarthritis affecting other joints. Ann Rheum Dis. 2014;73(9):1659-1664

2. Gellhorn AC, Katz JN, Suri P. Osteoarthritis of the spine: the facet joints. Nat Rev Rheumatol. 2013;9(4):216-224.

3. Hunter DJ, Bierma-Zeinstra S. Osteoarthritis. Lancet. 2019;393(10182):1745-1759.

4. Block JA. Osteoarthritis: OA guidelines: improving care or merely codifying practice? Nat Rev Rheumatol. 2014;10(6):324-326

5. Nelson AE, Allen KD, Golightly YM, Goode AP, Jordan JM. A systematic review of recommendations and guidelines for the management of osteoarthritis: the chronic osteoarthritis management initiative of the U.S. bone and joint initiative. Semin Arthritis Rheum. 2014;43(6):701-712.

6. Conaghan PG, Cook AD, Hamilton JA, Tak PP. Therapeutic options for targeting inflammatory osteoarthritis pain. Nat Rev Rheumatol. 2019;15(6):355-363.

7. Martel-Pelletier J, et al. Osteoarthritis. Nat Rev Dis Primers. 2016;2:16072.

8. Lories RJ, Luyten FP. The bone-cartilage unit in osteoarthritis. Nat Rev Rheumatol. 2011;7(1):43-49.

9. Aigner T, Söder S, Gebhard PM, McAlinden A, Haag J. Mechanisms of disease: role of chondrocytes in the pathogenesis of osteoarthritis--structure, chaos and senescence. Nat Clin Pract Rheumatol. 2007;3(7):391-399.

10. Pitsillides AA, Beier F. Cartilage biology in osteoarthritis--lessons from developmental biology. Nat Rev Rheumatol. 2011;7(11):654-663.

11. Hamerman D. The biology of osteoarthritis. N Engl J Med. 1989;320(20):1322-1330.

12. Hunter DJ. Pharmacologic therapy for osteoarthritis--the era of disease modification. Nat Rev Rheumatol. 2011;7(1):13-22.

13. Brandt KD, Radin EL, Dieppe PA, van de Putte L. Yet more evidence that osteoarthritis is not a cartilage disease. Ann Rheum Dis. 2006;65(10):1261-1264.

14. National Clinical Guideline Centre (UK). Osteoarthritis: Care and Management in Adults. London, United Kingdom: National Institute for Health and Care Excellence; 2014

15. Goldring SR, Goldring MB. Changes in the osteochondral unit during osteoarthritis: structure, function and cartilage-bone crosstalk. Nat Rev Rheumatol. 2016;12(11):632-644.

16. $\mathrm{Hu} \mathrm{X}$, et al. Cdc42 is essential for both articular cartilage degeneration and subchondral bone deterioration in experimental osteoarthritis. J Bone Miner Res. 2018;33(5):945-958.

17. Findlay DM, Atkins GJ. Osteoblast-chondrocyte interactions in osteoarthritis. Curr Osteoporos Rep. 2014;12(1):127-134.

18. Burr DB, Gallant MA. Bone remodelling in osteoarthritis. Nat Rev Rheumatol. 2012;8(11):665-673.

19. Findlay DM. Vascular pathology and osteoarthritis. Rheumatology (Oxford). 2007;46(12):1763-1768.

20. Burr DB. The importance of subchondral bone in the progression of osteoarthritis. J Rheumatol Suppl. 2004;70:77-80.

21. Burr DB. The importance of subchondral bone in osteoarthrosis. Curr Opin Rheumatol. 1998;10(3):256-262.

22. Mazur CM, et al. Osteocyte dysfunction promotes osteoarthritis through MMP13-dependent suppression of subchondral bone homeostasis. Bone Res. 2019; 7:34.

23. Alliston T, Hernandez CJ, Findlay DM, Felson DT, Kennedy OD. Bone marrow lesions in osteoarthritis: what lies beneath J Orthop Res. 2018;36(7):1818-1825.

24. Mansell JP, Tarlton JF, Bailey AJ. Biochemical evidence for altered subchondral bone collagen metabolism in osteoarthritis of the hip. Br J Rheumatol. 1997;36(1):16-19.

25. Mansell JP, Bailey AJ. Abnormal cancellous bone collagen metabolism in osteoarthritis. J Clin Invest. 1998;101(8):1596-1603.

26. Bailey AJ, Mansell JP, Sims TJ, Banse X. Biochemical and mechanical properties of subchondral bone in osteoarthritis. Biorheology. 2004;41(3-4):349-358

27. Mansell JP, Collins C, Bailey AJ. Bone, not cartilage, should be the major focus in osteoarthritis. Nat Clin Pract Rheumatol. 2007;3(6):306-307.

28. Suri S, Gill SE, Massena de Camin S, Wilson D, McWilliams DF, Walsh DA. Neurovascular invasion at the osteochondral junction and in osteophytes in osteoarthritis. Ann Rheum Dis. 2007;66(11):1423-1428. 
29. Walsh DA, et al. Angiogenesis and nerve growth factor at the osteochondral junction in rheumatoid arthritis and osteoarthritis. Rheumatology (Oxford). 2010;49(10):1852-1861.

30. Fransès RE, McWilliams DF, Mapp PI, Walsh DA. Osteochondral angiogenesis and increased protease inhibitor expression in OA. Osteoarthr Cartil. 2010;18(4):563-571.

31. Bonnet CS, Walsh DA. Osteoarthritis, angiogenesis and inflammation. Rheumatology (Oxford). 2005;44(1):7-16.

32. Lu J, et al. Positive-feedback regulation of subchondral H-type vessel formation by chondrocyte promotes osteoarthritis development in mice. J Bone Miner Res. 2018;33(5):909-920.

33. Saito M, et al. Angiogenic activity of subchondral bone during the progression of osteoarthritis in a rabbit anterior cruciate ligament transection model. Osteoarthr Cartil. 2012;20(12):1574-1582.

34. Cui Z, et al. Halofuginone attenuates osteoarthritis by inhibition of TGF- $\beta$ activity and H-type vessel formation in subchondral bone. Ann Rheum Dis. 2016;75(9):1714-1721.

35. Xie L, et al. Systemic neutralization of TGF- $\beta$ attenuates osteoarthritis. Ann N Y Acad Sci. 2016;1376(1):53-64.

36. Bertuglia A, Lacourt M, Girard C, Beauchamp G, Richard H, Laverty S. Osteoclasts are recruited to the subchondral bone in naturally occurring post-traumatic equine carpal osteoarthritis and may contribute to cartilage degradation. Osteoarthr Cartil. 2016;24(3):555-566.

37. Hayami T, et al. The role of subchondral bone remodeling in osteoarthritis: reduction of cartilage degeneration and prevention of osteophyte formation by alendronate in the rat anterior cruciate ligament transection model. Arthritis Rheum. 2004;50(4):1193-1206.

38. Karsdal MA, et al. Should subchondral bone turnover be targeted when treating osteoarthritis? Osteoarthr Cartil. 2008;16(6):638-646.

39. Edwards JR, Mundy GR. Advances in osteoclast biology: old findings and new insights from mouse models. Nat Rev Rheumatol. 2011;7(4):235-243.

40. Wu M, Chen W, Lu Y, Zhu G, Hao L, Li YP. Ga13 negatively controls osteoclastogenesis through inhibition of the AktGSK3ß-NFATc1 signalling pathway. Nat Commun. 2017;8:13700.

41. Guo Y, et al. Succinate and its G-protein-coupled receptor stimulates osteoclastogenesis. Nat Commun. $2017 ; 8: 15621$.

42. Drissi H, Sanjay A. The multifaceted osteoclast; far and beyond bone resorption. J Cell Biochem. 2016;117(8):1753-1756.

43. Park JH, Lee NK, Lee SY. Current understanding of RANK signaling in osteoclast differentiation and maturation. Mol Cells. 2017;40(10):706-713.

44. Chen W, Zhu G, Jules J, Nguyen D, Li YP. Monocyte-specific knockout of C/ebpa results in osteopetrosis phenotype, blocks bone loss in ovariectomized mice, and reveals an important function of $\mathrm{C} / \mathrm{ebp} \alpha$ in osteoclast differentiation and function. $J$ Bone Miner Res. 2018;33(4):691-703.

45. Boyce BF. Advances in the regulation of osteoclasts and osteoclast functions. J Dent Res. 2013;92(10):860-867.

46. Xie H, et al. PDGF-BB secreted by preosteoclasts induces angiogenesis during coupling with osteogenesis. Nat Med. 2014;20(11):1270-1278.

47. Andrae J, Gallini R, Betsholtz C. Role of platelet-derived growth factors in physiology and medicine. Genes Dev. 2008;22(10):1276-1312.

48. Cao R, et al. Angiogenic synergism, vascular stability and improvement of hind-limb ischemia by a combination of PDGF-BB and FGF-2. Nat Med. 2003;9(5):604-613

49. Zhang J, Cao R, Zhang Y, Jia T, Cao Y, Wahlberg E. Differential roles of PDGFR-alpha and PDGFR-beta in angiogenesis and vessel stability. FASEB J. 2009;23(1):153-163.

50. Ostendorf T, Boor P, van Roeyen CR, Floege J. Platelet-derived growth factors (PDGFs) in glomerular and tubulointerstitial fibrosis. Kidney Int Suppl (2011). 2014;4(1):65-69.

51. Jitariu AA, Raica M, Cîmpean AM, Suciu SC. The role of PDGF-B/PDGFR-BETA axis in the normal development and carcinogenesis of the breast. Crit Rev Oncol Hematol. 2018;131:46-52.

52. Kusumbe AP, Ramasamy SK, Adams RH. Coupling of angiogenesis and osteogenesis by a specific vessel subtype in bone. Nature. 2014;507(7492):323-328.

53. Ramasamy SK, Kusumbe AP, Itkin T, Gur-Cohen S, Lapidot T, Adams RH. Regulation of hematopoiesis and osteogenesis by blood vessel-derived signals. Annu Rev Cell Dev Biol. 2016;32:649-675.

54. Mapp PI, Walsh DA. Mechanisms and targets of angiogenesis and nerve growth in osteoarthritis. Nat Rev Rheumatol. 2012;8(7):390-398.

55. Walsh DA, Bonnet CS, Turner EL, Wilson D, Situ M, McWilliams DF. Angiogenesis in the synovium and at the osteochondral junction in osteoarthritis. Osteoarthr Cartil. 2007;15(7):743-751.

56. Fang H, et al. Early changes of articular cartilage and subchondral bone in the DMM mouse model of osteoarthritis. Sci Rep. 2018;8(1):2855.

57. Wang Y, et al. TNF- $\alpha$-induced LRG1 promotes angiogenesis and mesenchymal stem cell migration in the subchondral bone during osteoarthritis. Cell Death Dis. 2017;8(3):e2715.

58. Filipowska J, Tomaszewski KA, Niedźwiedzki Ł, Walocha JA, Niedźwiedzki T. The role of vasculature in bone development, regeneration and proper systemic functioning. Angiogenesis. 2017;20(3):291-302.

59. Sufen G, Xianghong Y, Yongxia C, Qian P. bFGF and PDGF-BB have a synergistic effect on the proliferation, migration and VEGF release of endothelial progenitor cells. Cell Biol Int. 2011;35(5):545-551.

60. Zhu S, et al. Subchondral bone osteoclasts induce sensory innervation and osteoarthritis pain. J Clin Invest. 2019;129(3):1076-1093.

61. Walsh DA, Hu DE, Mapp PI, Polak JM, Blake DR, Fan TP. Innervation and neurokinin receptors during angiogenesis in the rat sponge granuloma. Histochem J. 1996;28(11):759-769.

62. Glasson SS, Blanchet TJ, Morris EA. The surgical destabilization of the medial meniscus (DMM) model of osteoarthritis in the 129/SvEv mouse. Osteoarthr Cartil. 2007;15(9):1061-1069.

63. Cobos EJ, Ghasemlou N, Araldi D, Segal D, Duong K, Woolf CJ. Inflammation-induced decrease in voluntary wheel running in mice: a nonreflexive test for evaluating inflammatory pain and analgesia. Pain. 2012;153(4):876-884 